\title{
La technologie de débitage laminaire bipolaire au Proche- Orient durant le Néolithique précéramique B (PPNB)
}

\author{
Ferran Borrell \\ Consejo Superior de Investigaciones Científicas (CSIC-IMF). Egipcíaques 15, 08001, Barcelona, Espagne. \\ Email: silmarils1000@hotmail.com
}

\section{Résumé :}

Cet article présente un état des lieux actualisé des origines, de la diffusion et de la variabilité de la technologie laminaire bipolaire durant le Néolithique précéramique B dans l'aile occidentale du Croissant fertile. Les recherches confirment que son apparition aux alentours de 8900-8800 cal. BCE dans la moyenne vallée de l'Euphrate marque un changement décisif dans les traditions lithiques locales et la technologie des projectiles. Sa large diffusion dans des contextes socio-économiques variés, utilisant des matières premières différentes, et sa variabilité régionale et spatiale croissante observée dans le Levant suggèrent que la technologie bipolaire n’a, à priori, pas exigée l'existence d'une infrastructure économique soutenant une forme de spécialisation artisanale. Il apparaît alors essentiel d'évaluer si, et dans quelles circonstances, la technologie bipolaire peut être considérée comme une production spécialisée. Le débat sur les conditions de diffusion de cette technologie laminaire (transfert de connaissances ou "colonisation » démographique) est loin d'être clos. Son expansion rapide à travers différents écosystèmes ainsi que son adoption tout aussi rapide par des communautés fondées sur différents modes de subsistance suggère que la diffusion de la technologie laminaire bipolaire ne constitue qu'un élément d'une diffusion culturelle majeure plutôt que la simple expansion d'une méthode de débitage efficace. Enfin, l'abandon de cette technologie s'est produit dans des circonstances et des chronologies différentes au sein du Levant, ce qui révèle un processus de " déconnexion » entre le nord et le sud du Levant, entre les différents écosystèmes du Proche-Orient et des divergences croissantes dans les développements culturels du Néolithique dans la région commençant entre 7200 et $6900 \mathrm{cal}$. BCE.

Mots-clés : Proche-Orient; Néolithique précéramique B; industries lithiques; naviforme; technologie laminaire bipolaire

\section{Introduction et contexte chronoculturel}

La technologie laminaire bipolaire (TLB) est traditionnellement considérée comme la composante la plus commune des traditions de taille de silex du Néolithique précéramique $B$ (PPNB) au Levant durant les $9^{\text {ème }}$ et $8^{\text {ème }}$ millénaires cal. BCE (Figure 1 et Tableau 1 ). La première définition explicite de cette production laminaire à partir d'un nucléus à plans de frappe opposé nous vient de J. Cauvin (1968). Il inventa le terme «naviforme » en raison de la forme générale des nucléus étudiés. Leurs dos, façonnés par enlèvements bifaciaux,

Published by the School of History, Classics and Archaeology, University of Edinburgh ISSN: 2055-0472. URL: http://journals.ed.ac.uk/lithicstudies/

This work is licensed under a Creative Commons Attribution 2.5 UK: Scotland License. 
forment une crête (en forme de quille de navire) et donnent ainsi au nucléus une forme similaire à celle d'un bateau. Un plan de frappe est établi à chaque extrémité d'un nucléus en silex ou obsidienne qui en général, a fait l'objet, au préalable, d'un façonnage bifacial l'inscrivant dans une morphologie ovale ou trapézoïdale. L'exploitation de la surface de débitage à partir des deux plans de frappe est entièrement complémentaire et ne se limite pas à des corrections ponctuelles des enlèvements provenant de l'un des plans de frappe. Cette exploitation bipolaire offre une maitrise sans précédent de la configuration des lames que le tailleur produit, ce qui permet d'obtenir une régularité dans la production qui apparaît impossible avec d’autres technologies de débitage laminaire par percussion.

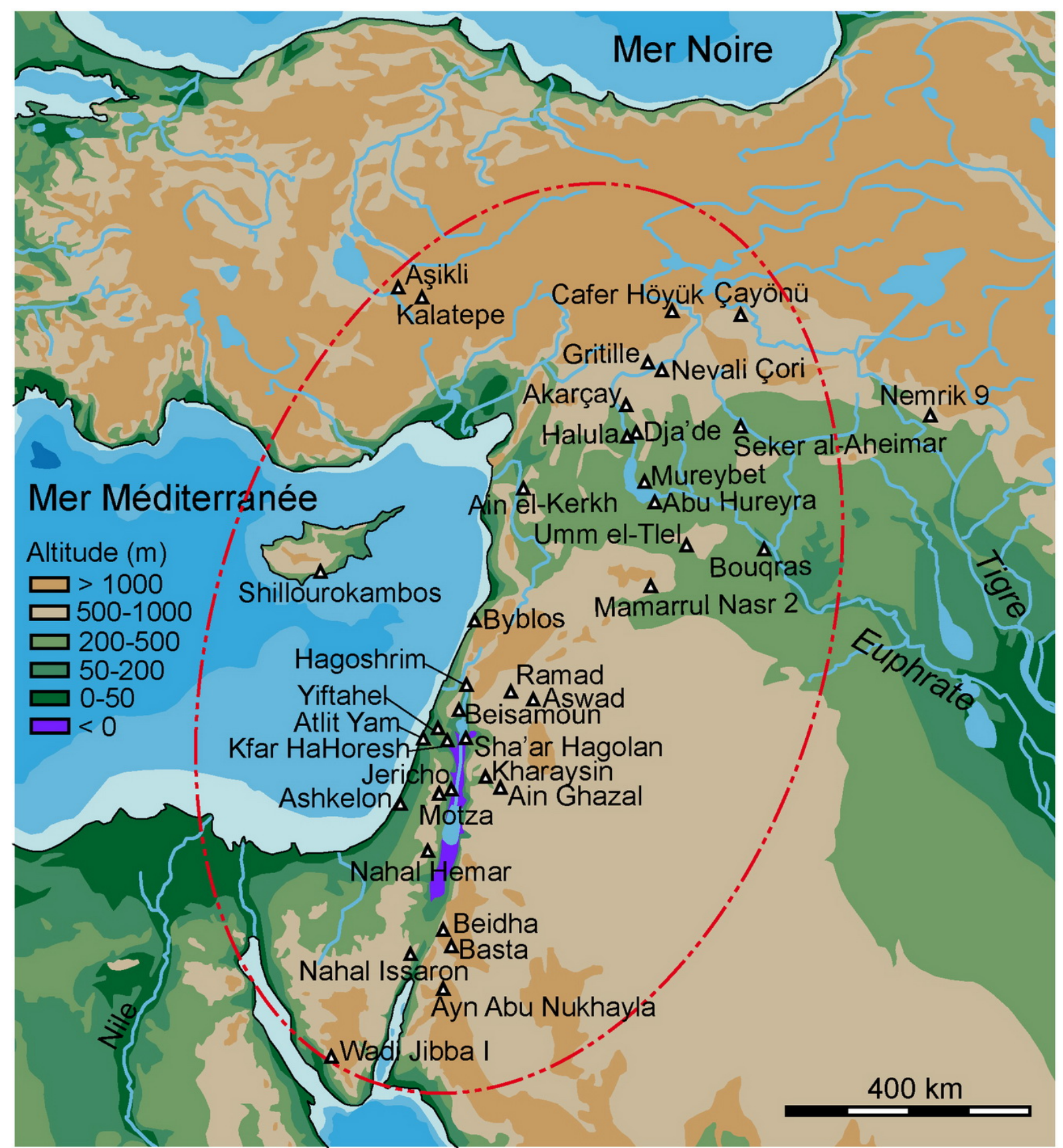

Figure 1. Expansion géographique approximative de la TLB durant le PPNB et sélection des principaux sites néolithiques levantins.

Figure 1. Approximate geographical expansion of BBT during the PPNB and a selection of major Neolithic sites in the Levant. 
Tableau 1. Chronologie approximative proposée pour le Néolithique précéramique au Levant.

Table 1. Approximate chronology proposed for the Pre-Pottery Neolithic in the Levant.

\begin{tabular}{lclc}
\hline $\begin{array}{l}\text { Levant nord } \\
\text { (Aurenche } \text { et } \text { al. 1981) }\end{array}$ & cal. BCE & $\begin{array}{l}\text { Levant sud } \\
\text { (Goring-Morris et al. 2009) }\end{array}$ & cal. BCE \\
\hline PPNA & $10200-8800$ & PPNA & $9600-8500$ \\
PPNB ancien & $8800-8200$ & PPNB ancien & $8500-8100$ \\
PPNB moyen & $8200-7600$ & PPNB moyen & $8100-7500$ \\
PPNB récent & $7600-6900$ & PPNB récent & $7500-6750$ \\
Néolithique céramique (PPNB final) & $6900-6400$ & PPNB final-PPNC & $6750-6400$ \\
& & Néolithique céramique & $6400-5600$ \\
\hline
\end{tabular}

Le débitage est orienté vers la production de larges lames centrales très standardisées, généralement robustes, rectilignes, naturellement pointues, et obtenues alternativement à partir de chacun des deux plans de frappe (Figure 2). Ces lames centrales ciblées, aussi connues sous le nom de lames prédéterminées (Perrot 1968: 395; Abbès 2003), ont été principalement transformées en grandes pointes de projectiles (par exemple, les types Byblos, Amuq, Ugarit et Jéricho) bien que secondairement d'autres outils aient été confectionnés à partir de ces mêmes lames (par exemple, des éléments de faucille, des couteaux, grattoirs ou burins) (Figures 3 et 4). Les économies de débitage nous apprennent que toutes sortes d'outils ont aussi été produit à partir d'une large gamme de sous-produits allant de gros éclats corticaux à des lames latérales rectilignes (lames débordantes) détachés au cours de cette séquence de débitage. Cette terminologie a ensuite été adoptée par de nombreux chercheurs et finalement, le terme naviforme est devenu synonyme de la technologie laminaire bipolaire au Levant (par exemple, Nishiaki 1994; 2000; Quintero \& Wilke 1995).

Suite à sa définition initiale, et en raison du développement ultérieur des études lithiques dans ces régions, la TLB a fait l'objet d'études intensives et exhaustives menées par différentes écoles et selon des approches diverses (Abbès 2003; Arimura 2007; Baird 1994; Barzilai 2010; Borrell 2011c; Calley 1986a; J. Cauvin 1968; 1994; Crowfoot-Payne 1984; Kozłowski 1999; Nishiaki 2000; Quintero \& Wilke 1995; Wilke \& Quintero 1994; 2010), contribuant toutes à une meilleure compréhension de la vaste gamme d'aspects socioéconomiques des premiers systèmes agricoles du Levant. Dans certains cas, les approches se sont orientées vers une compréhension de la distribution temporelle et spatiale de la technologie bipolaire dans tout le Levant (Bader 1989; Bar-Yosef 1981; Barzilai 2010; Borrell \& Khalaily 2016; Crowfoot-Payne 1983), tandis que d'autres approches ont mis l'accent sur la pertinence socio-économique et culturelle de cette technologie au cours du PPNB en tant que moyen de définir différentes régions culturelles (par exemple, la province levantine ou le Moyen Euphrate) (Cauvin \& Cauvin 1993; Kozłowski 1999; Kozłowski \& Aurenche 2005: 76), où sphères d'interaction (Borrell \& Molist 2014). Diverses analyses technologiques se sont focalisées, non seulement sur la reconstitution de la chaîne opératoire de cette méthode caractéristique de débitage laminaire, mais aussi sur la détermination des compétences technologiques et des comportements de ses utilisateurs (par exemple, Abbès 1998; 2003; Abbès \& Deraprahamian 2002; Barzilai 2010; Borrell 2011c; Calley 1986a; Nishiaki 2000) en incorporant, dans certains cas, des travaux expérimentaux (Quintero \& Wilke 1995; Wilke \& Quintero 1994). De telles analyses ont permis d'identifier différentes variantes chronologiques et régionales de cette technologie au cours du PPNB à travers le Levant. Elles ont aussi démontré que le terme « naviforme » était trop restrictif pour définir la variabilité observée à la fois dans la préparation des nucléus et dans l'organisation des séquences de débitage. 

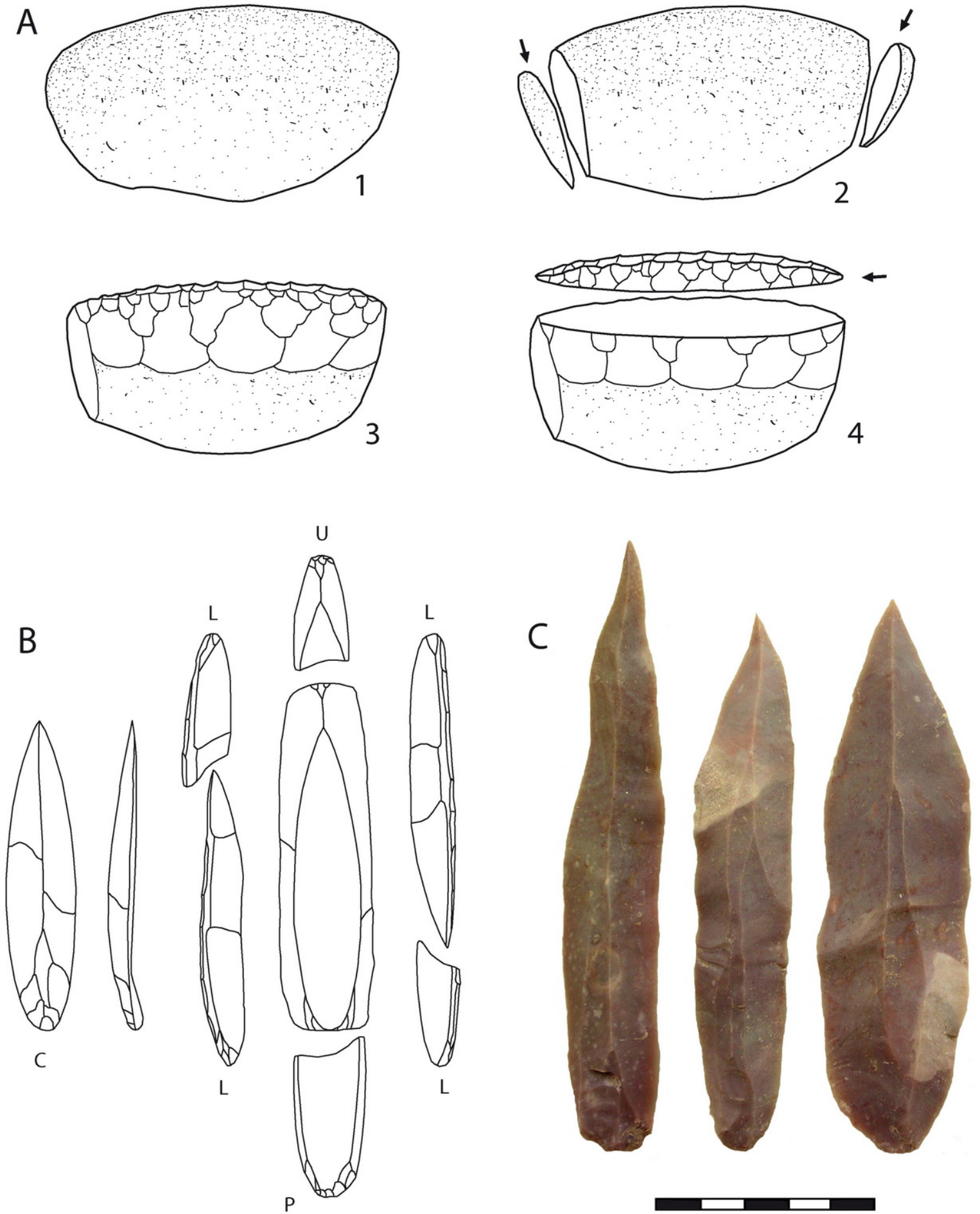

Figure 2. Préparation d'un nucléus à lames bipolaires à crête frontale (A); exemple de l'un des schémas de séquence de débitage et de ses produits les plus diagnostiques (c: lame centrale, u : lame de reprise distale [upsilon], 1 : lame latérale et p : reprise proximale) (B); trois lames centrales non-retouchées d'Halula (cache de trois lames produites à l'extérieur du site) (C). Dessins A et B d’après Abbès (2003: 182, 187). La barre d'échelle mesure $5 \mathrm{~cm}$ de long, avec des segments de $1 \mathrm{~cm}$.

Figure 2. Preparation of a bidirectional blade core with a frontal ridge (A); An example of one of the schemes of the reduction sequence and its most diagnostic products (c: central blade, u: reprise distal [upsilon], l: lateral blade and p: reprise proximal) (B); three unretouched central blades from Halula (cache of three blades produced off-site) (C). Drawings A and C from Abbès (2003: 182, 187). The scale bar is 5 cm long, with 1 cm segments. 

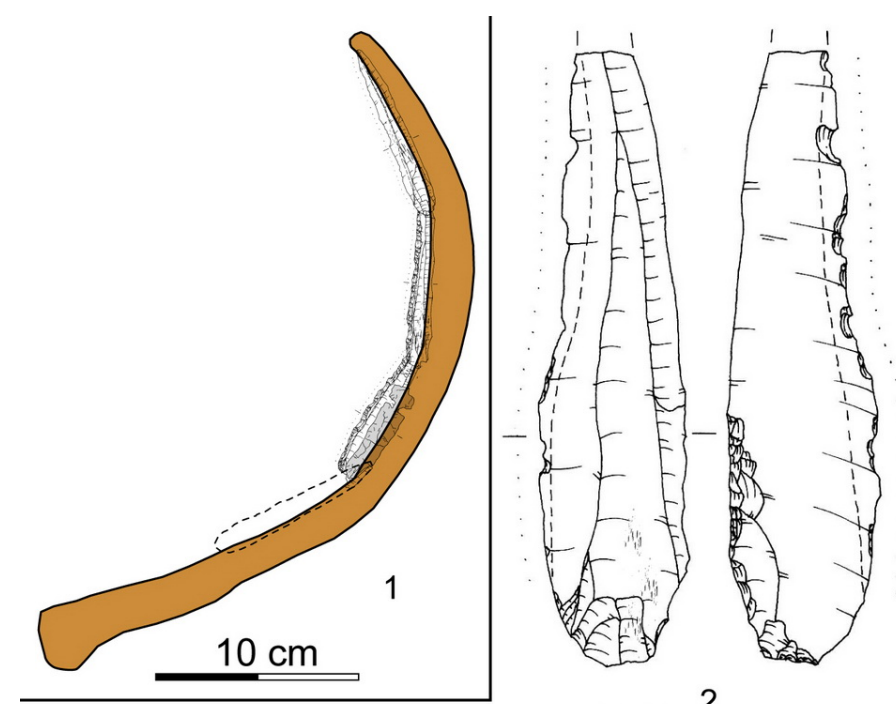

2
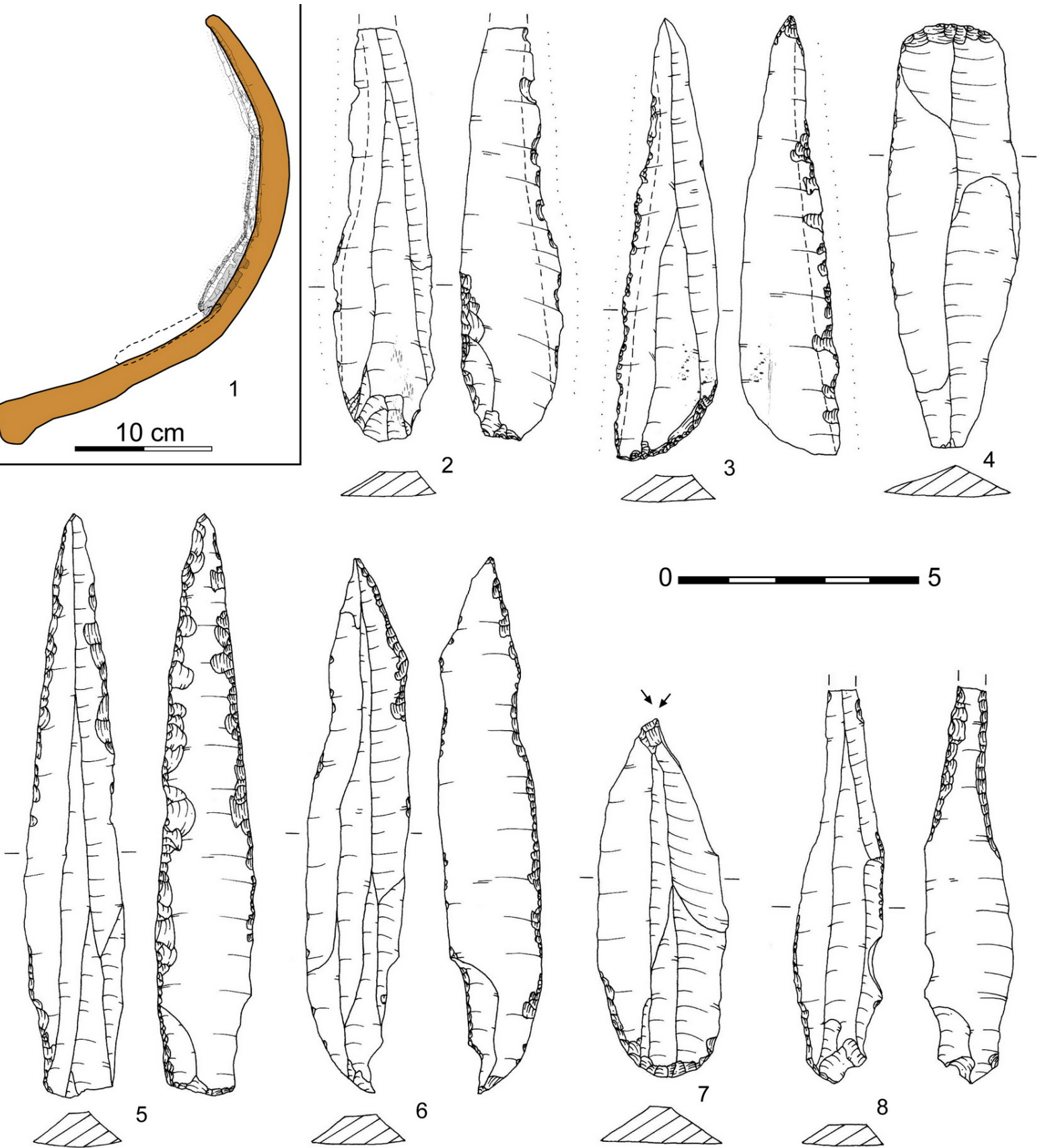

8

Figure 3. Reconstitution d'une faucille d'Halula (PPNB récent) avec 4 lames faucilles (dont 3 complètes) fabriquées à partir de lames centrales bipolaires (1) et outils fabriqués sur lames bipolaires ; éléments de faucille (2 et 3), un grattoir (4), des couteaux (5 et 6), un burin dièdre (7) et un perçoir (8). La barre d'échelle mesure 5 $\mathrm{cm}$ de long, avec des segments de $1 \mathrm{~cm}$.

Figure 3. Reconstruction of a sickle from Halula (Late PPNB) with four sickle blades (3 complete) made from central bidirectional blades (1) and other tools made on bidirectional blades: sickle blades (2 and 3), a scraper (4), knives (5 and 6), a dihedral burin (7) and a borer (8). The scale bar is $5 \mathrm{~cm}$ long, with $1 \mathrm{~cm}$ segments.

Cet article présente une évaluation de l'état actuel de nos connaissances sur les origines de la technologie laminaire bipolaire et sa diffusion rapide dans tout le Levant. Il délivre en second lieu un résumé des différentes variantes régionales et temporelles des schémas de débitage bipolaires identifiées dans ces régions, à partir des études antérieures fondées essentiellement sur la typologie des nucléus et des approches technologiques plus récentes visant à mieux comprendre la chaîne opératoire complète de cette méthode de débitage laminaire. Cette étude approfondie permettra, espérons-le, d’atténuer la potentielle confusion 
terminologique et conceptuelle causée par l'abondance des études provenant des différentes écoles et approches.
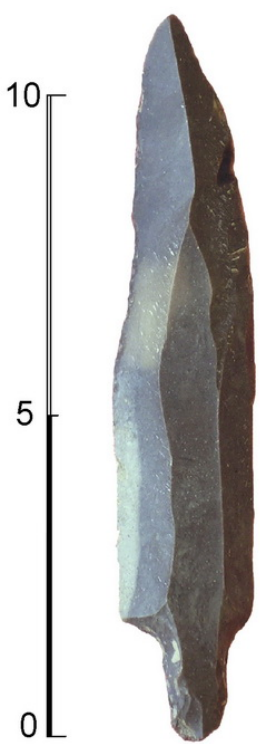

1

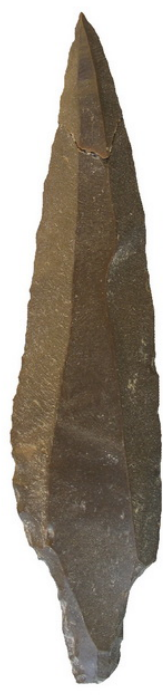

2



3

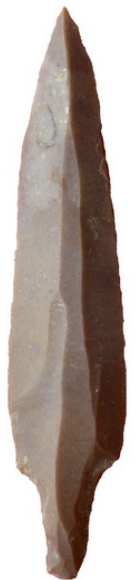

4

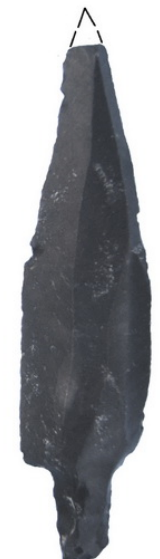

5



6

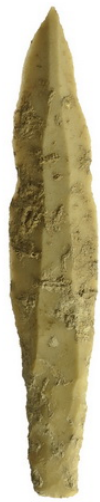

7


8

Figure 4. Pointes de Byblos d'Halula (1-4) et d'Akarçay (5), une pointe de Jéricho de Kharaysin (6) et pointes d'Amuq d'Yiftahel (7-9) toutes manufacturées sur des lames centrales. La barre d'échelle mesure $10 \mathrm{~cm}$ de long. Figure 4. Byblos points from Halula (1-4) and Akarçay (5), a Jericho point from Kharaysin (6) and Amuq points from Yiftahel (7-9), all made on bidirectional central blades. The scale bar is $10 \mathrm{~cm}$ long.

\section{La technologie de débitage laminaire bipolaire au Levant nord}

\subsection{Origines et précocité de la première diffusion}

Avec l'apparition et le développement de la culture PPNA (Néolithique précéramique A) au Levant, un changement majeur s'opère dans les technologies de production de l'outillage lithique. Le microlithisme caractéristique des périodes épipaléolithiques est progressivement abandonné, comme on l'observe à Hatoula et Mureybet, en faveur de la production de lames de plus en plus longues et larges (par exemple, Abbès 2003; Calley 1986a; M.C. Cauvin 1994; Lechevallier \& Ronen 1994; Valla \& Plisson 2005). La technologie laminaire bipolaire trouve ses origines dans le nord du Levant (moyenne vallée de l'Euphrate) à la fin du PPNA récent et au début du PPNB ancien, autour du premier quart du $9^{\text {ème }}$ millénaire cal. BCE sur des sites tels que Cheikh Hassan, Jerf el Ahmar et Mureybet IIIB-IVA (Abbès 1998; 2003; 2007; Calley 1986a; Stordeur \& Abbès 2002). Elle s’est progressivement développée à partir d'une technologie de débitage laminaire unipolaire à deux plans de frappe opposés documentée au cours du PPNA (Mureybet IIIA et Jerf el Ahmar) - l'un de deux plans de frappe étant uniquement destiné à corriger les bords distaux des lames détachées à partir du plan de frappe opposé.

A partir du milieu du $9^{\text {ème }}$ millénaire cal. BCE la TLB diffuse le long de la moyenne vallée de l'Euphrate jusqu'à des sites tels que Dja'dé (phase 1) (Coqueugniot 1994), Nevalı Çori (Schmidt 1994) et Göbekli (Layer 2) (Schmidt 2001; Dietrich 2016) ainsi que vers le nord-ouest de la Syrie (vallée de l'Oronte), à Ain el-Kerkh (Layers 7-9) (Tsuneki et al. 2006). Des indices d'une production in situ de lames centrales ou prédéterminées ont été reportés dans chacun de ces sites (présence de nucléus naviformes, de lames upsilon, de tablettes, de lames à crête et de pointes de Byblos). Sa dispersion rapide a continué vers l'Ouest, pour atteindre Chypre aux alentours de 8500 cal. BCE où une technologie de débitage laminaire bipolaire orientée vers la production de larges pointes (non pas à partir de nucléus 
« naviformes », mais d'une variante à crête postéro-latérale) apparaît à Shilourokambos (début de la phase A) (Briois et al. 2007; 2013).

La diffusion de la TLB vers les vallées supérieures de l'Euphrate et du Tigre a suivi un processus plus complexe. Une proportion significative de lames bipolaires et d'outils finis (pointes de Byblos), souvent obtenus à partir de nucléus "naviformes » standardisés, a été reporté lors de l'étude des industries lithiques appartenant aux sous-phases «Grill and Channeled Building » (étape lithique 2) de Çayönü (ca. 8500-8100 cal. BCE) (Caneva et al. 2001). La présence de telles lames et outils finis à Çayönü a été interprétée comme une preuve de réseaux complexes d'échanges intercommunautaires, vraisemblablement originaires de la moyenne vallée de l'Euphrate (Binder 2008). Ce n’est que vers 8000 cal. BCE que la présence abondante de pointes de Byblos, de nucléus «naviformes » et des éléments indicatifs de leur débitage apparaissent à Çayönü (sous-phases «Cobble et Cell Buildings », [étape lithique 3]) (Binder 2008; Caneva et al. 2001) et à Cafer (phase ancienne) (Cauvin 1989), indiquant que la technologie bipolaire a été complètement adoptée et intégrée aux traditions lithiques locales des hautes vallées de l'Euphrate et du Tigre. C'est aussi durant cette période que l'obsidienne est incorporée comme la principale matière première utilisée pour la production de lames bipolaires, en complément de son utilisation pour la production de lames et lamelles par pression.

Vers le milieu du $8^{\text {è̀me }}$ millénaire cal. BCE la TLB constitue la base des industries de pierre taillée dans la plupart, sinon la totalité des sites datés de la fin du PPNB moyen et du PPNB récent au Levant nord (par exemple, Halula, Akarçay, Hayaz Höyük, Gritille, Bouqras, Abu Hureyra, Ain el-Kerkh, etc.). Elle devient ainsi la composante la plus commune de la culture matérielle lithique de la région. Les lames bipolaires sont alors produites à partir de l'obsidienne et d'une large gamme de silex, de grain moyen à grain fin (locaux ou importés) provenant à la fois de gîtes primaires et secondaires (terrasses de rivières et d'oueds).

\subsection{Les variantes au cours du PPNB}

Dans ses travaux majeurs sur les technologies lithiques du PPNB dans le nord de la Syrie, Abbès (1998; 2003) conclu que les nucléus "naviformes » tels que décrits par Cauvin (1968) ne représentent qu'une variante de trois modes distincts de gestion des blocs initiaux et de configuration des nucléus (préformes) et de deux modalités différentes d'extraction de lames "prédéterminées » en superposition et en juxtaposition. Il propose alors d'introduire le terme plus approprié de " systèmes bipolaires " (Abbès 2003). Abbès utilise également le terme " prédéterminé » pour désigner les lames centrales ciblées par les intentions du débitage (voulues, de première intention) mettant ainsi l'accent sur le fait que l'ensemble de la chaîne opératoire est intentionnellement organisé dans le but de produire les lames pointues caractéristiques mentionnées ci-dessus. Les trois variantes de configuration des nucléus et de gestion des blocs initiaux incluent : la modalité «bipolaire » (préforme avec une seule crête frontale centrée) (Figures 5A et 6A); la modalité "postéro-latérale » (préforme avec une crête frontale centrée et une crête dorsale postéro-latérale) (Figures 5B et 6B) ; et la modalité " naviforme " (préforme avec une crête frontale et une crête dorsale, toutes deux centrées) (Figures 5C et 7). Les différences initiales dans les configurations des nucléus ne semblent pas influencer leur exploitation ultérieure et n'affectent que faiblement le plein débitage des nucléus. En outre, aucune de ces variantes dans la préforme des nucléus ne semble être restreinte à une région ou une chronologie particulière du Levant. 



A

B

C
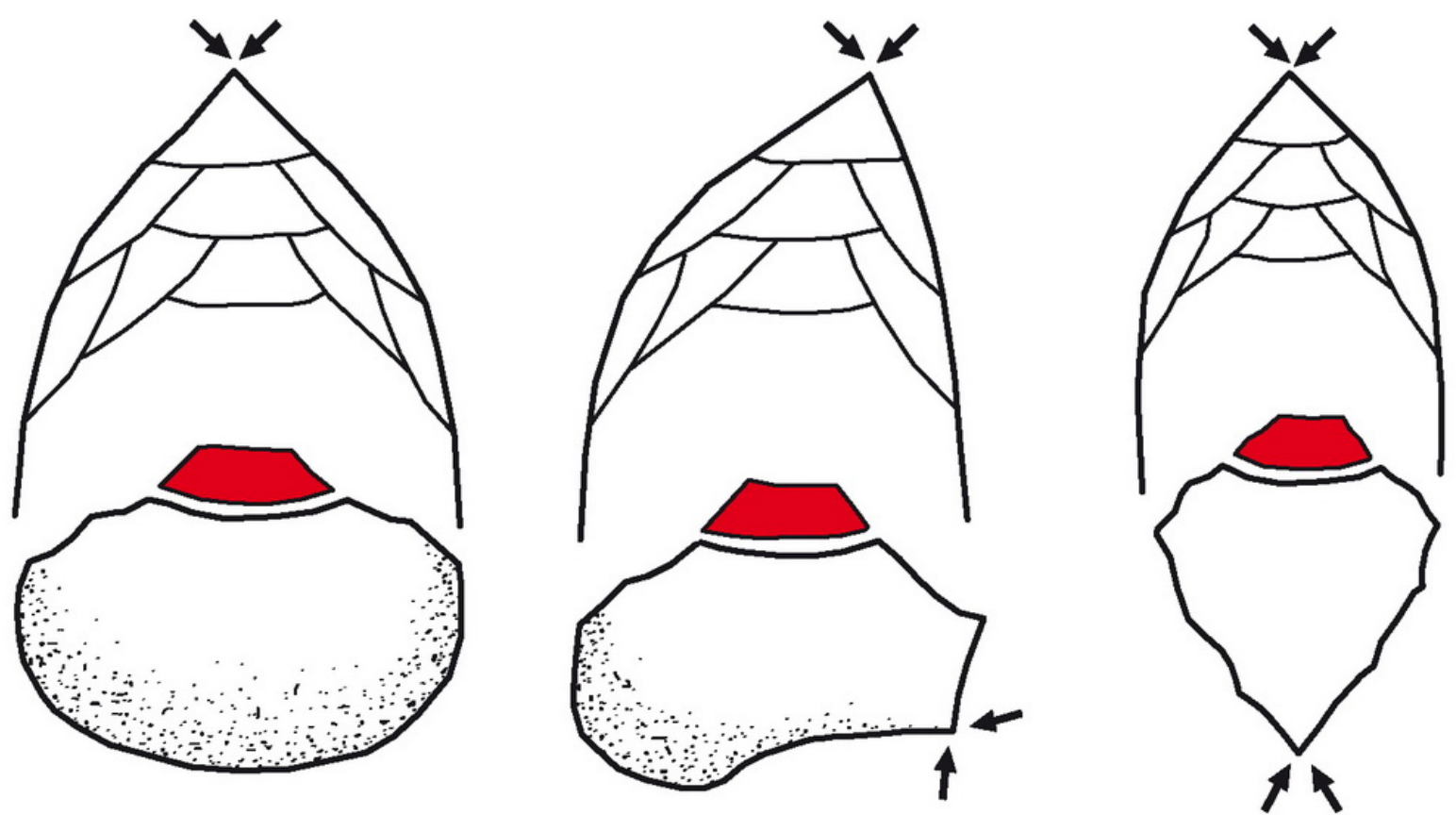

Figure 5. Nucléus bipolaire préformé avec une crête frontale centrée (A) ; une crête frontale centrée et une crête dorsale postéro-latérale (B) ; et une crête frontale et une crête dorsale, toutes deux centrées (naviforme) (C) (Abbès 2003: 178).

Figure 5. Bidirectional cores preformed with a frontal crested ridge (A); a frontal crested ridge and a posterolateral dorsal ridge (B); and with a frontal and dorsal crested ridge ('naviform') (C) (Abbès 2003: 178).

La reconstitution de la chaîne opératoire des débitages bipolaires à Mureybet et Cheikh Hassan (en observant l'agencement des négatifs des enlèvements à la fois sur les lames centrales et sur les surfaces de débitage des nucléus) a également permis de différencier deux modalités d'extraction de lames prédéterminées en superposition. La première est caractéristique du PPNB ancien (chaque séquence d'exploitation complète du nucléus est composée d’1 lame "prédéterminée » + 2 larges lames latérales distales [appelées débordantes] + 2 petites lames ou lamelles de correction proximale). La seconde correspond au début (aux phases initiales) du PPNB moyen (Figure 2C) (1 lame "prédéterminée" + 2 à 4 lames débordantes +1 lame de correction proximale +1 lame de correction distale 
[«upsilon »] + d'autres lames de correction proximale ou distale + quelques lames ou lamelles d'entretien secondaire de la surface de débitage). Cette dernière modalité est le schéma ou la variante la plus répandue dans le Levant à partir du PPNB moyen jusqu'à son abandon. Certaines modifications mineures de ces schémas rigides ont aussi été identifiées par l'auteur (Abbès 2003: 184-186). D’autres études ont également indiqué que l'exploitation de nucléus larges (environ $6 \mathrm{~cm}$ de largeur) s’opère selon des schémas moins rigides comme on peut l'observer au sein des niveaux du PPNB récent d'Akarçay et du PPNB final d'Umm el Tlel (Borrell 2011a; Borrell et al. 2013).
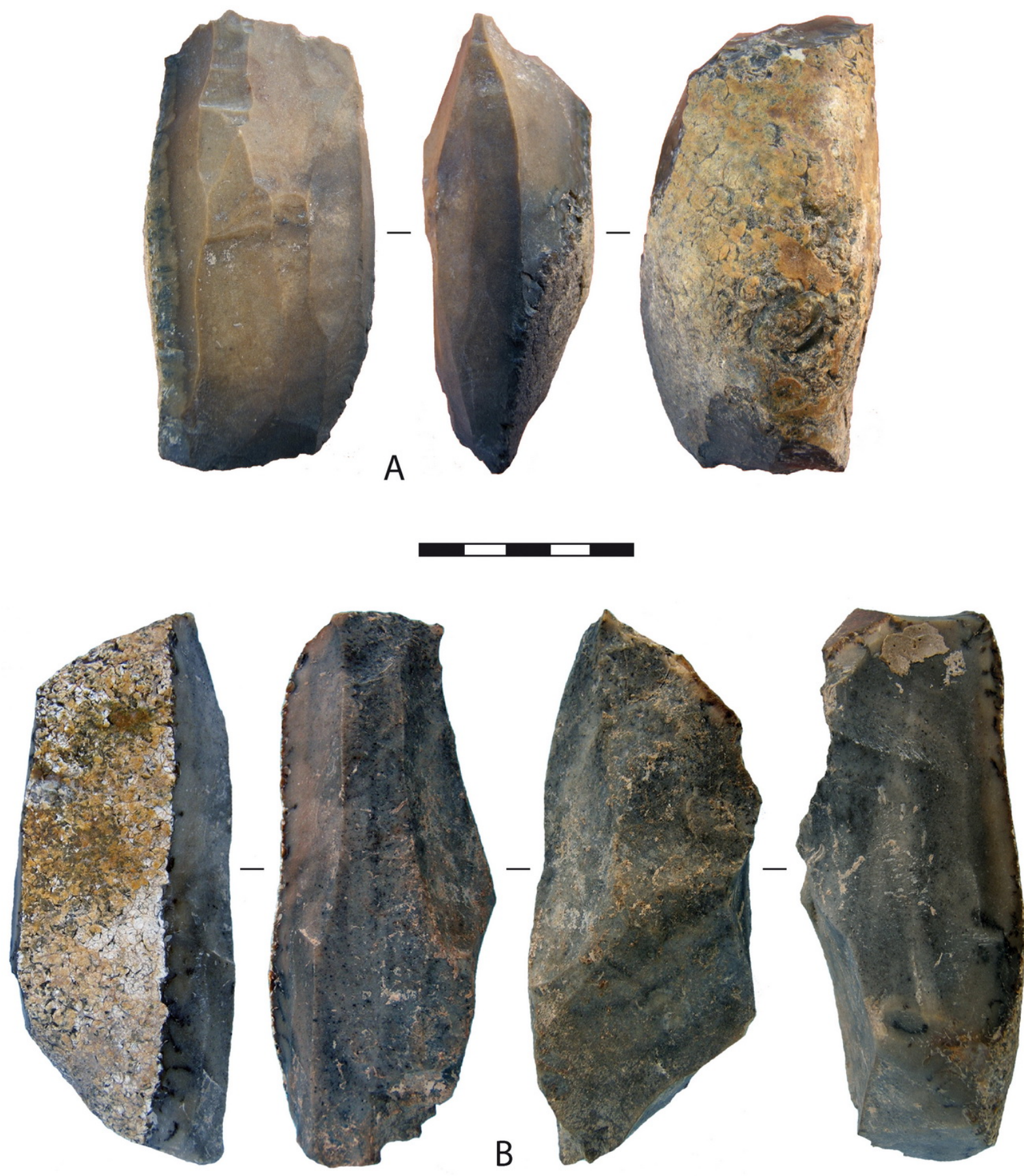

Figure 6. Exemples de nucléus bipolaires des niveaux du PPNB récent d'Akarçay (Turquie) : avec un dos naturel (A) et une crête dorsale postéro-latérale (B). La barre d'échelle mesure $5 \mathrm{~cm}$ de long, avec des segments de $1 \mathrm{~cm}$. Figure 6. Examples of bidirectional cores from the Late PPNB layers from Akarçay (Turkey): with an unmodified back (A) and with a postero-lateral ridge (B). The scale bar is $5 \mathrm{~cm}$ long, with $1 \mathrm{~cm}$ segments. 

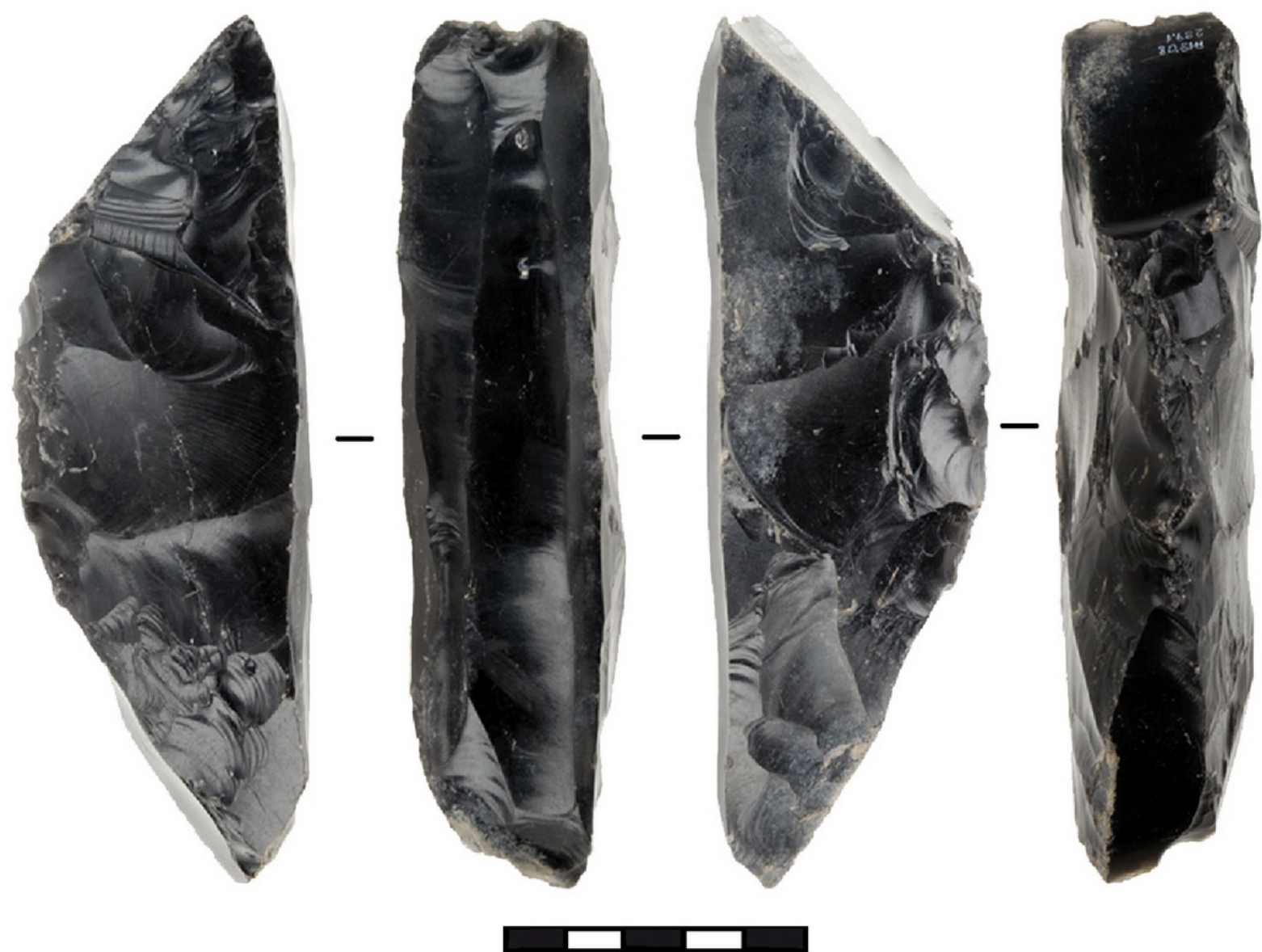

Figure 7. Exemple d'un nucléus «naviforme » (en obsidienne) de Kayırl1-Bitlikeler, Anatolie Centrale (photo: Berkay Dinçer) (Balcı 2013). La barre d'échelle mesure $5 \mathrm{~cm}$ de long, avec des segments de $1 \mathrm{~cm}$.

Figure 7. Example of a 'naviform' core (of obsidian) from Kayırl1-Bitlikeler, Central Anatolia (photo: Berkay Dinçer) (Balc1 2013). The scale bar is $5 \mathrm{~cm}$ long, with $1 \mathrm{~cm}$ segments.

Les techniques de débitages impliquées dans la TLB ont été déterminées par différents analystes concluant que les lames étaient détachées par percussion directe, après une abrasion du plan de frappe, en utilisant un percuteur en pierre tendre (Abbès 2003; 2008; Abbès \& Déraprahamian 2002; Quintero \& Wilke 1995) ; aucune différence chronologique ou spatiale n’a été identifiée à cet égard. Les mêmes études indiquent également que les nucléus sont maintenus dans la main durant le débitage, et que le détachement des lames s’opère jusqu’à ce que les nucléus présentent des dimensions trop faibles pour être maintenu efficacement. Cependant, certains auteurs suggèrent, bien que l'hypothèse n’ait pas été développée de manière plus approfondie, que, dans certains cas d'exploitation très poussées, les nucléus ont pu être maintenus à l'aide d’un système d'immobilisation (Abbès \& Déraprahamian 2002).

\subsection{La stratégie bipolaire " off-set "}

Une variante régionale très particulière de la TLB a été identifiée à la fin des années 1970 (Akazawa 1979) et 1980 (Fujii 1986) dans la région du désert syrien central. Elle a été décrite dans les années 1990 sous le nom de «méthode naviforme de type Douara » (Nishiaki 1994; 2000) et récemment redéfinie et renommée "stratégie bipolaire " off-set " » après avoir été largement documentée dans la moyenne vallée de l’Euphrate (Borrell 2011c). Cette variante se caractérise par une surface de débitage étroite et torse et par la manière spécifique de l'exploiter (Figures 8-10). L'un des éléments caractéristiques de cette stratégie d'exploitation est la possibilité de débiter des lames centrales ciblées et épaisses sans avoir nécessairement 
recours au détachement de lames «upsilon » ou de lames latérales droites. La direction du débitage est légèrement décalée par rapport à l'axe longitudinal du nucléus, elle s’étend du bord droit d'un premier plan de frappe jusqu'au bord opposé du plan de frappe distal du nucléus. Les nervures de la dernière lame centrale sont utilisées comme si il s'agissait d'un négatif d'enlèvement de lame latérale, pour la production de la lame centrale suivante qui sera détachée à partir du plan de frappe opposé. La gestion du volume est conçue de cette manière afin d’obtenir un ratio élevé de production de lames centrales standardisées et pointues, bien que légèrement torses, sans agrandir pour autant les préformes par rapport aux nucleus naviforme " classique ». Les lames et nucléus offrent des traits hautement diagnostiques (par exemple, la partie proximale des lames est déjetée vers la droite et la face inférieure présente généralement un négatif d'enlèvement provenant du bord droit, en partie proximale et destiné à retirer intentionnellement le talon et le bulbe. Grâce à ces caractéristiques diagnostiques, il a été possible de définir la distribution spatio-temporelle de cette variante, constituant un techno-complexe spécifique, dans le Levant nord (Borrell 2011c; 2013).

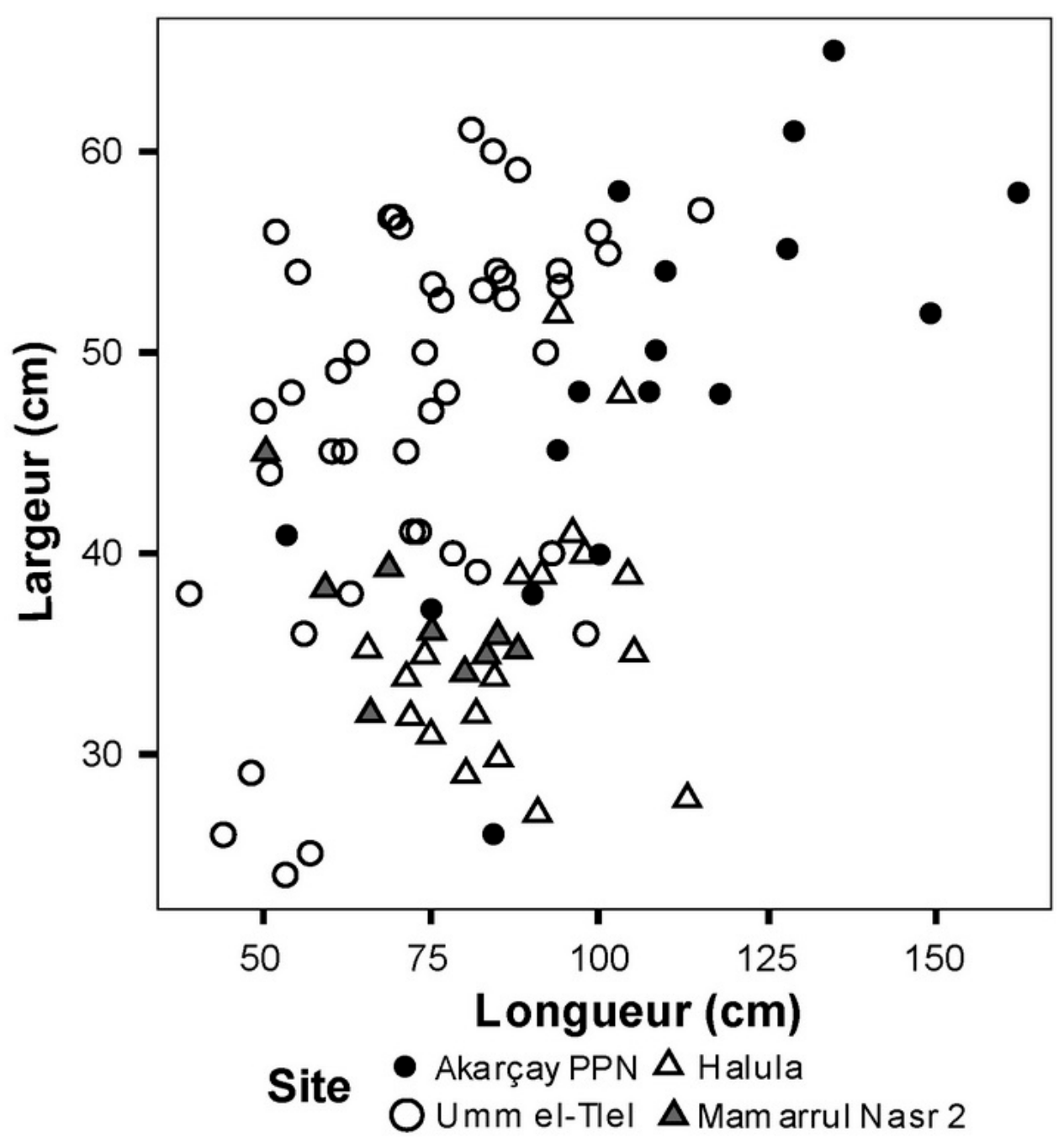

Figure 8. Comparaison des indices de longueur et largeur des nucléus bipolaires « off-set » de Halula (moyenne vallée de l'Euphrate, Syrie) et Mamarrul Nasr 2 (bassin de Douara, Syrie) avec les nucléus bipolaires d'Akarçay (sud-est de la Turquie) et d’Umm el Tlel (désert syrien central). Notez l’homogénéité délivrée par les nucléus bipolaires « off-set » courts et étroits par rapport aux nucléus bipolaires plus larges d'Akarçay et Umm el Tlel. Figure 8. Comparison of the length and width indexes of the 'off-set' bidirectional cores from Halula (middle Euphrates valley, Syria) and Mamarrul Nasr 2 (Douara basin, Syria) with the bidirectional cores from Akarçay (south-east Turkey) and Umm el Tlel (central Syrian Desert). Note the homogeneity showed by the narrow and short 'off-set' cores when compared to the wide bidirectional cores from Akarçay and Umm el Tlel. 




Figure 9. Diagramme de la séquence de débitage bipolaire « off-set » (pour plus d’informations voir Borrell 2011c). La barre d'échelle mesure $5 \mathrm{~cm}$ de long, avec des segments de $1 \mathrm{~cm}$.

Figure 9. Diagram of the reduction 'off-set bidirectional strategy' knapping sequence (for details see Borrell 2011c). The scale bar is $5 \mathrm{~cm}$ long, with $1 \mathrm{~cm}$ segments. 




A


C



$\mathrm{E}$
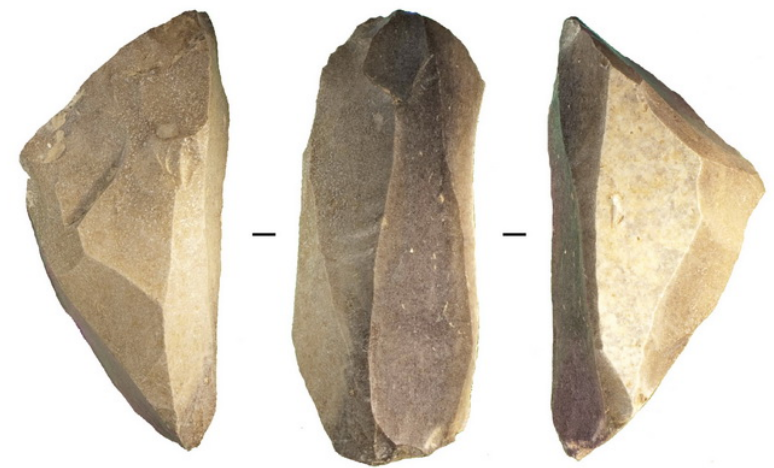

B
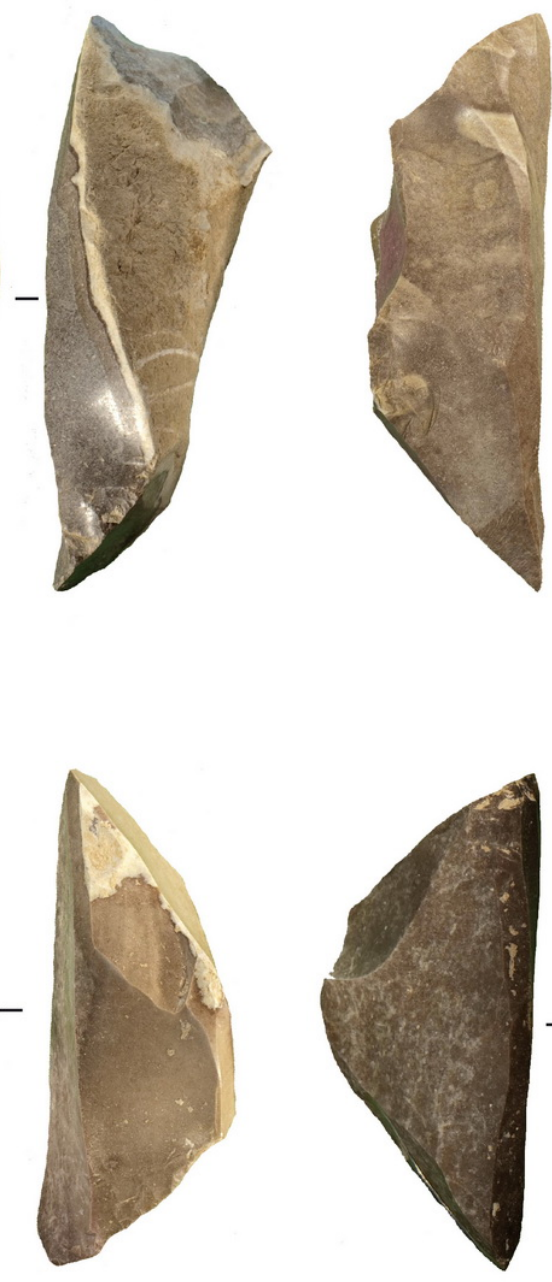

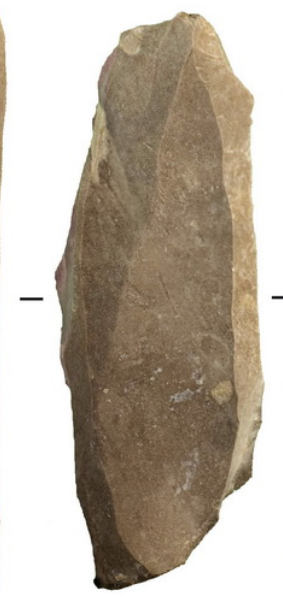

D
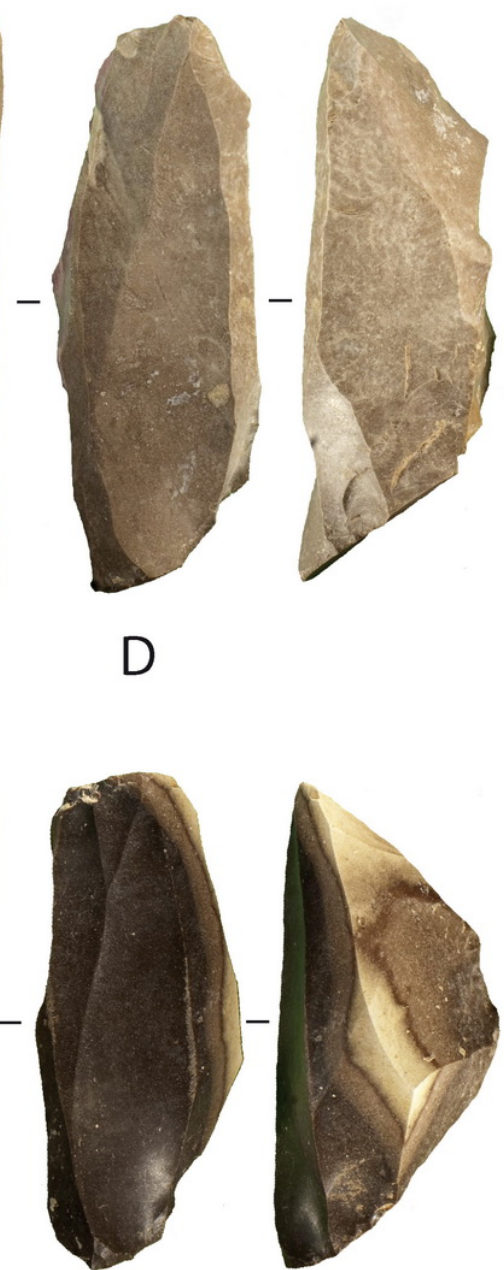

$\mathrm{F}$

Figure 10. Exemples de nucléus bipolaires «off-set» provenant de diverses localités au sein du complexe néolithique de Mamarrul Nasr (Borrell 2011c). La barre d'échelle mesure $5 \mathrm{~cm}$ de long, avec des segments de 1 $\mathrm{cm}$.

Figure 10. Examples of 'off-set' bidirectional cores from different locations from the Neolithic complex of Mamarrul Nasr (Borrell 2011c). The scale bar is $5 \mathrm{~cm}$ long, with $1 \mathrm{~cm}$ segments.

L’utilisation intensive de la stratégie bipolaire " off-set » est attestée dans deux régions principales ; la partie inférieure du moyen Euphrate (dans les grands villages agricoles tels que Halula, Abu Hureyra et Bouqras) et dans le désert syrien central (au sein d'ateliers de 
débitage et de campements tels que Douara Cave 2 et Mamarrul Nasr 2). Les produits (lames centrales) manufacturés dans ces régions nucléaires ont été, comme pour les autres types de bipolaire, principalement transformés en pointes de projectiles. Ces produits ont circulé jusque dans la partie supérieure de la moyenne vallée de l'Euphrate (Akarçay), ainsi que dans la vallée du Balikh (Sabi Abyad et Gürçütepe), la vallée du Khabur (Seker al-Aheimar) et la vallée de l'Oronte (Ain el-Kerkh 2) sous la forme d'échanges n'ayant pas entrainé de transfert de cette stratégie bipolaire « off-set » à ces régions.

En résumé, la stratégie bipolaire « off-set » a prospéré dans la vallée de l’Euphrate à partir d'environ 7800 jusqu'à 7100 cal. BCE. Dans le désert central syrien sa date d'apparition est encore débattue (elle est soit contemporaine à la vallée de l'Euphrate, soit plus tardive, aux alentours de 7000-6500 cal. BCE) (pour plus de détails voir Borrell 2011c; 2013; Borrell et al. 2011; 2013). Les comparaisons avec la TLB de sites plus anciens de la moyenne vallée de l'Euphrate et des régions avoisinantes (Dja'de, Mureybet, Jerf el-Ahmar, Cheikh Hassan, Abr 3, Qaramel, Göbekli, Nevalı Çori, etc.) ne délivrent aucune filiation avec l'émergence ultérieure de la stratégie bipolaire «off-set». Son apparition doit donc être interprétée comme issue d'un développement régional prenant place entre 8000 et $7800 \mathrm{cal}$. BCE.

\subsection{Le déclin et la disparition de la technologie bipolaire}

L'abandon progressif de la TLB dans le nord du Levant débute à partir du dernier quart du $8^{\text {ème }}$ millénaire cal. BCE (fin du PPNB récent) (Borrell 2011a; Borrell \& Khalaily 2016). L'analyse des assemblages lithiques de deux établissements majeurs dans la vallée de l'Euphrate (Halula et Akarçay) indique qu'une simplification des technologies de production lithique s'engage aux alentours de 7300-7200 cal. BCE et affecte l'ensemble du processus de production: les stratégies d'approvisionnement en matières premières, les méthodes et techniques de débitages et les types d'outils retouchés. Le débitage laminaire diminue rapidement en faveur d'une production d'éclats et, au sein même de ces débitages laminaires, la technologie bipolaire décroît au profit de modalités d'exploitation unipolaires. Cette transition d'une production laminaire à une production d'éclats est attestée dès les dernières étapes du PPNB Récent, clairement avant l'apparition des premières poteries dans la région aux alentours de 7000-6900 cal. BC (Nishiaki \& Le Mière 2005) et se poursuit durant le Néolithique céramique. Un processus similaire est observé dans la haute vallée du Tigre à Çayönü lors des dernières étapes de la séquence précéramique (la sous-phase « Large Room ») (Caneva et al. 2001). La TLB persiste en tant que méthode de débitage secondaire pendant les premiers stades du Néolithique céramique (Pré-Halaf) comme on l'observe à Halula et Akarçay (Borrell 2011a; 2011b; 2015) mais elle est aussi signalée dans d'autres établissement du Néolithique céramique nouvellement fondés comme à Shir (RokittaKrumnow 2011). Il faut noter que le désert central syrien (les bassins de Douara, d'El Kowm et de Palmyre) suivent un développement culturel très différent de celui des vallées des fleuves plus au nord. La technologie bipolaire reste la méthode principale utilisée pour la production laminaire au moins jusqu'au milieu du $7^{\text {ème }}$ millénaire cal. BCE avec parfois de nouvelles modalités de percussion (Abbès 2003; Borrell \& Khalaily 2016; Calley 1986b).

\section{La technologie bipolaire au Levant sud}

\subsection{Variantes et diffusion}

La technologie laminaire bipolaire a été initialement identifiée dès la seconde moitié du $9^{\text {ème }}$ millénaire cal. BCE au sein de quelques rares sites datés du PPNB ancien dans le Levant sud (région boisée méditerranéenne) comme Motza (level VI), Horvat Galil et Kfar HaHoresh 
(Barzilai 2010; 2013; Khalaily et al. 2007). Les études des niveaux PPNB ancien de Motza (Khalayli et al. 2007) ont confirmé les hypothèses proposées pour Horvat Galil (Gopher 1997) qui suggéraient qu'au cours de ces premiers stades, la technologie bipolaire ne représentait qu'une partie marginale des assemblages lithiques locaux principalement orientés vers des technologies laminaires unipolaires et une production d'éclats. La présence quasi exclusive de lames centrales ciblées et d'outils retouchés sur ces sites ainsi que l'absence virtuelle de nucléus bipolaires (seulement 1 pièce sur 41 identifiables pour un total de 60 pièces à Motza et 3 pièces sur 28 identifiables pour un total de 56 nucléus à Horvat Galil) et d'éléments de préparation ou de réfection de nucléus suggèrent un débitage mené à l'extérieur des sites ou une importation des lames (à partir du Levant nord) (Barzilai 2013; Gopher 1997). L'étude de la cache de lames de Motza qui a abouti au remontage de sept agrégats (Barzilai 2010; 2013; Khalaily et al. 2007), a révélé un schéma de débitage particulier dénommé « one-on-one " (Figure 11A). Cette variante " one-on-one », dont la distribution spatiale et chronologique dans le Levant sud est encore peu connue, a été interprété comme un développement non local, attribué hypothétiquement au Levant nord en raison de quelques similitudes partagées avec les industries de la fin du PPNA de Mureybet (Barzilai 2013). Ce schéma consiste en une extraction alternative de lames pointues (en série) à partir des deux plans de frappe opposés. L'extraction de ces lames permet d'exploiter $90 \%$ de la surface de débitage sans recours nécessaire à une maintenance (pas besoin de lames «upsilon » par exemple). Il est intéressant de noter que cette variante " one-on-one » semble être associée à l'exploitation d'un silex rose luisant de très bonne qualité mécanique principalement utilisé pour la production de pointes d'Helwan (Barzilai 2013).

La TLB remplaça rapidement les traditions lithiques locales. Des preuves d'une production in situ ont été signalées presque simultanément dans différentes régions de la moitié septentrionale du Levant sud : 1) dans le sud de la Syrie au cours du dernier quart du $9^{\text {ème }}$ millénaire cal. BCE (par exemple, Qarassa North (phases 1 et 2) (Communications personnelles avec J.J. Ibáñez en 2017) et Tell Aswad (phase moyenne) (Stordeur et al. 2010), 2) en Galilée durant la transition $9^{\text {ème }}-8^{\text {ème }}$ millénaire au sein d'une série de sites nouvellement fondés tels que Yiftahel, Nahal Zippori 3, Ahihud (Garfinkel et al. 2012; Caracuta et al. 2014; J. Vardi, communication personnelle), et 3) dans le centre-ouest de la Jordanie à 'Ain Ghazzal (phases PPNB moyen 1 et 2) (Rollefson et al. 1992) et Kharaysin (Ibáñez et al. 2015). Durant cette période, le schéma de production bipolaire "predetermined-upsilon » a remplacé le schéma " one-on-one » (Barzilai 2010) (Figure 11B). La variante "predetermined-upsilon » est la plus largement documentée dans la région. Elle correspond approximativement aux deux modalités d'extraction de lames prédéterminées superposées (les modalités PPNB ancien et moyen) définies par Abbès (2003) dans la moyenne vallée de l'Euphrate. La reconstitution de ce schéma de débitage repose principalement sur les travaux antérieurs visant à restituer la séquence de débitage complète des nucléus naviformes (Quintero 2010; Quintero \& Wilke 1995; Wilke \& Quintero 1994). Dans ces études, les auteurs utilisent des termes différents tels que «Naviform Core-and-Blade Technology » ou " Naviform Blade Technology ». D’autres différences dans la terminologie utilisée (par exemple, l'usage du terme «intended blade» au lieu de "predetermined blade ») dérivent de divergences conceptuelles entre les auteurs. 


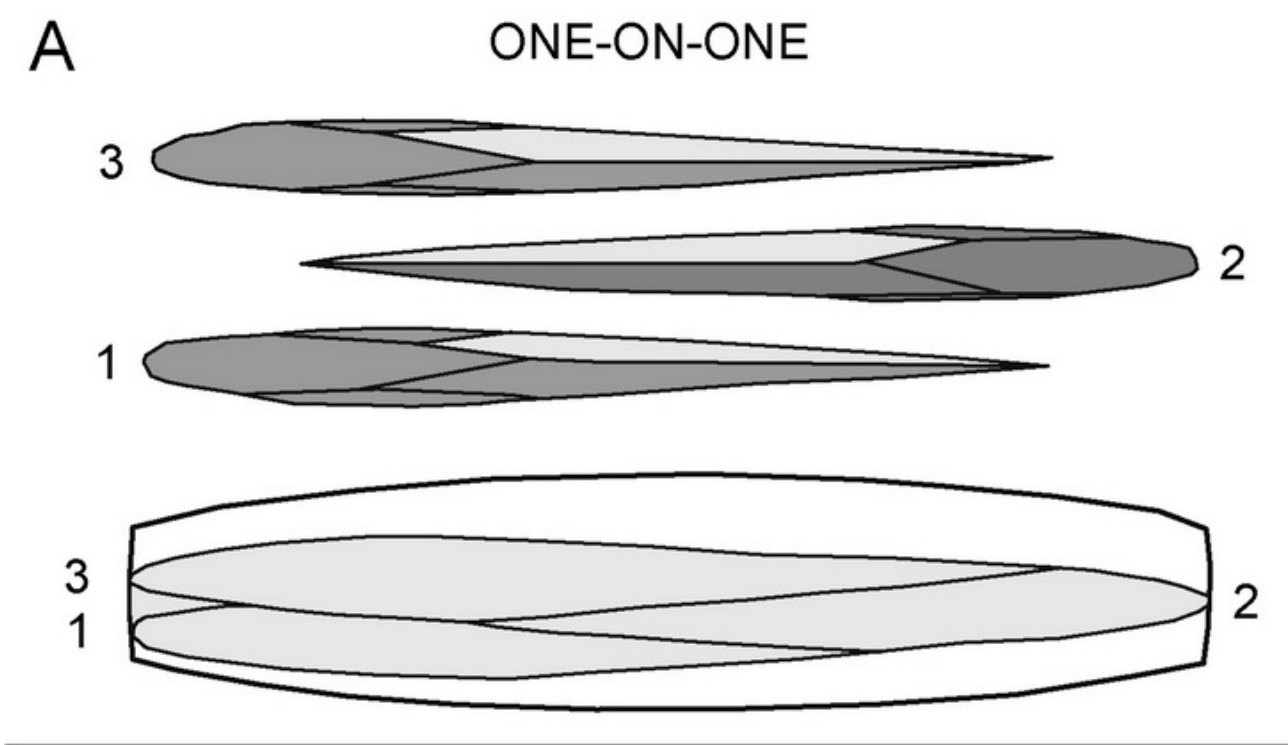

B PREDETERMINED-UPSILON

2
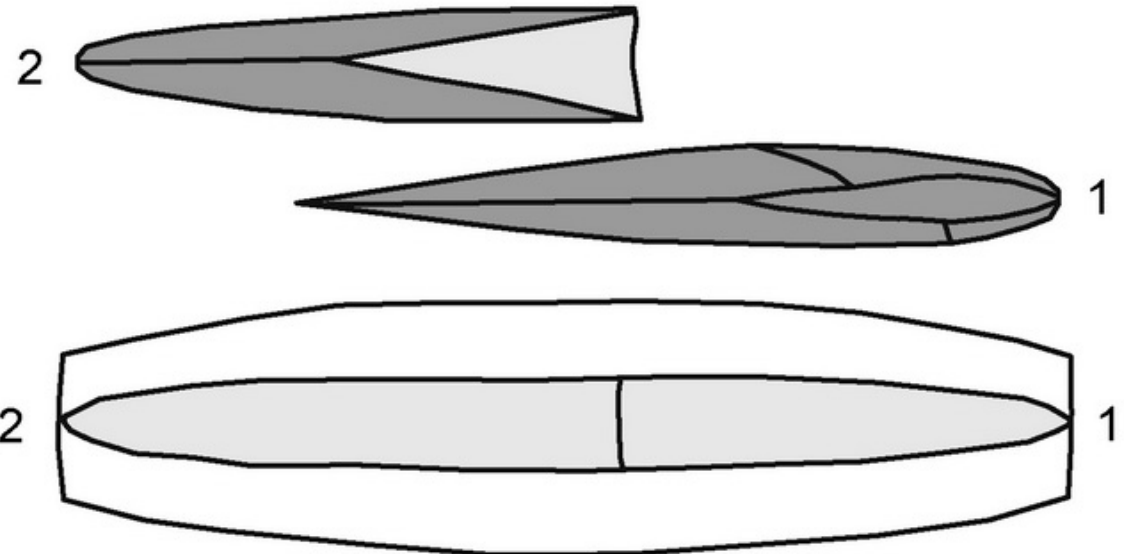

C SINGLE-DOMINANT PLATFORM

2
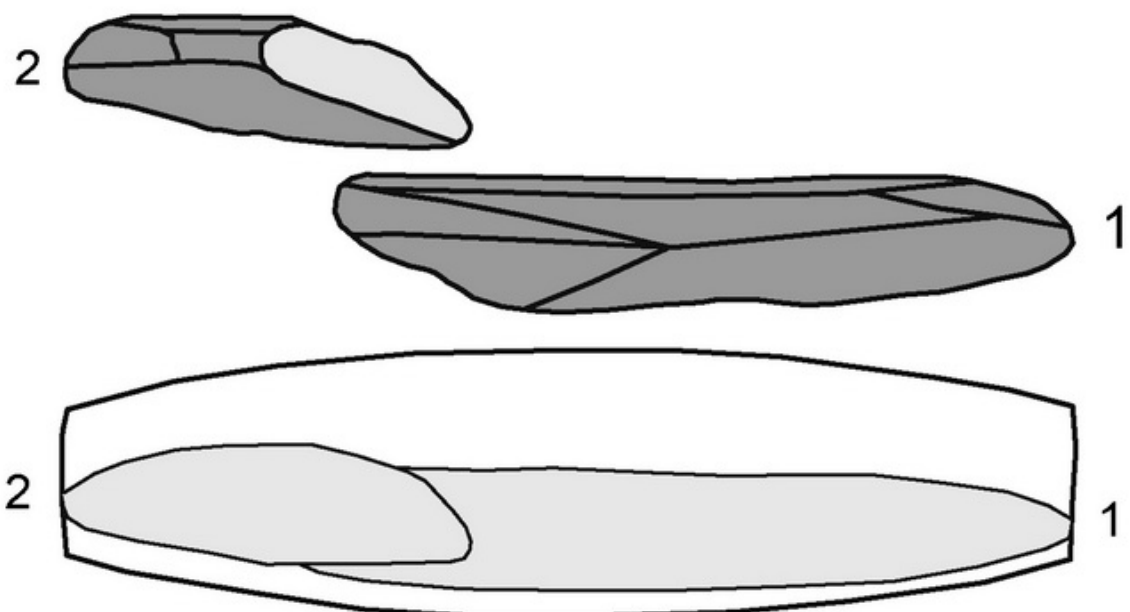

Figure 11. Les trois schémas bipolaires de débitage identifiés dans le Levant sud tel que définis par Barzilai (2013).

Figure 11. The three bidirectional knapping schemes identified in the southern Levant as defined by Barzilai (2013). 
Durant la première moitié du $8{ }^{\text {ème }}$ millénaire cal. BCE (PPNB moyen), La TLB devient la méthode de dominante pour la production de lames dans la grande majorité des sites du Levant sud, de la région boisée méditerranéenne (Abu Gosh, Jéricho, etc.) jusqu’au Néguev (Nahal Hemar, Nahal Issaron, etc.), au sud de la Transjordanie (Beidha et Ayn Abu Nukhayla) et au Sinaï (Wadi Tbeik et Wadi Jibba I). L'abondance des nucléus et d'éléments diagnostiques de la séquence de débitage, ainsi que l'utilisation intensive de matières premières locales indique, dans la plupart des cas, un débitage in situ. La majorité de l'outillage (pointes de projectiles, éléments de faucille, burins transverses, perçoirs et forêts, etc.) est fabriqué sur des lames bipolaires. La TLB atteint des niveaux élevés de savoir-faire (artisanat) où certains sites sont interprétés comme centres régionaux de production spécialisée (Yiftael et 'Ain Ghazzal par exemple) exportant une partie de leur production vers d’autres localités (Milevski et al. 2008). Le Néguev, en revanche, constitue l'exception à la règle puisque quelques auteurs proposent l'existence d'un système local différent : le «single dominant platform » (Figure 11C) (Barzilai 2013; Barzilai \& Goring-Morris 2011). La reconstitution de ce schéma de débitage, basée sur l'étude des remontages du site NL 1021 (Mitki et al. 2013), indique une variante adaptative et plus opportuniste où les blocs de silex ne sont généralement pas préformés et la séquence de débitage n’est pas aussi stricte qu'au sein des schémas ciblant l'obtention de lames centrales pointues. Les lames sont détachées à partir d'un plan de frappe, l'autre plan de frappe ne sert qu'à l'enlèvement occasionnel de produits d'entretien de la surface de débitage (Barzilai 2013). Aucune lame prédéterminée ou upsilon n'est produite au sein de ce schéma de débitage où la productivité laminaire est faible. $\mathrm{Au}$ regard de cette variante "single dominant platform » qui se caractérise par le fait que les lames sont débitées à partir d'un seul plan de frappe, il semble raisonnable de reconsidérer son appartenance à la définition stricte des systèmes bipolaires dans la région.

Le PPNB récent (durant la seconde moitié du $8^{\text {ème }}$ millénaire cal. BCE) est une période encore peu étudiée sur le littoral méditerranéen à l'ouest du Jourdain, en raison d'un manque de données. Cette période est, en revanche, bien représentée en Transjordanie, en particulier dans la vaste région de Petra (Gebel 2004). Dans cette région, au sein de la vallée du Rift et plus à l'est, seule la variante « upsilon-predetermined » est représentée (Barzilai 2013). Cette variante est également largement utilisée dans une grande partie du Levant sud au cours des dernières étapes du PPNB durant la première moitié du $7^{\text {ème }}$ millénaire cal. BCE (PPNB finalPPNC). On la retrouve sur des sites tels que Beisamoun et Ashkelon (Borrell \& Khalaily 2016) bien qu'elle soit déjà abandonnée dans d’autres régions comme la Transjordanie (Rollefson 1990; 2001).

Trois types de préparation de nucléus (avec une crête dorsale (centrée) ['naviforme'], avec une crête postéro-latérale et avec un dos non modifié cortical [bipolaire]) sont attestées dans le Levant sud, et coexistent dans certain cas, au PPNB (Barzilai 2013). Les différences chronologiques et régionales ne semblent pas suffisamment cohérentes, selon les connaissances actuelles, pour considérer la présence ou l'absence des divers types de nucléus comme marqueurs chronologiques.

\subsection{Le déclin et la disparition des technologies bipolaires}

Au début du Néolithique céramique, la production d'éclats constitue la base des industries de pierre taillées. Au sein d'une composante laminaire en déclin, les lames bipolaires constituent un pourcentage faible compris entre 10 et $20 \%$ des lames produites et les lames unipolaires (en percussion directe) prédominent comme sur les sites Yarmoukien de Sha'ar Hagolan (couche 3b, secteur N) (Barzilai \& Garfinkel 2006), Hagoshrim (Getzov 1999), Munhatta IIB (Gopher 1989), Hamadiya et Tel Roïm niveaux II-II-IV (Nadel \& Nadler-Uziel 2011). Un peu plus tard, la TLB est presque totalement abandonnée (comme, 
par exemple, à Sha’ar Hagolan-secteur E et Tel Te’o) (Barzilai \& Garfinkel 2006; Eisenberg et al. 2001; Matskevich 2011). Ces données suggèrent que l'abandon de la TLB dans cette partie du Levant se produit au cours de la transition PPNC-PPNB final au Néolithique céramique vers le milieu du $7^{\text {ème }}$ millénaire cal. BCE ou un peu plus tardivement (Borrell \& Kalaily 2016).

\section{La technologie bipolaire en Anatolie centrale}

Les premières études des industries lithiques d'Aşıklı, un site PPNB majeur près des abondantes sources d'obsidienne de Cappadoce, évoquent la présence de nombreux nucléus à lames à plans de frappe opposés et de pointes de Byblos, coexistant avec une production de microlithes sur le site (Balkan-Atl1 1994). Afin de documenter les premières étapes de production (et de diffusion) d'obsidienne ainsi que les méthodes et techniques de débitages utilisées dans la région, les recherches entreprises au milieu et à la fin des années 90 se sont orientées vers l'identification d'ateliers près des sources d'obsidienne (Balkan-Atlı et al. 1997; 1999). Une série d'ateliers de taille d'obsidienne datant du Néolithique ont été retrouvés à Golludağ est, Golludağ ouest et Nenezi Dağ. Les fouilles du vaste atelier multipériodes de Kümürcü-Kalatepe ont fourni une grande quantité de nucléus «naviformes » standardisés en obsidienne ainsi que des nucléus prismatiques unipolaires et des nucléus unipolaires à lames arquées (Binder 2002; Binder \& Balkan-Atl1 2001). Les produits des débitages laminaires bipolaires ont été, au moins partiellement exportés vers la moyenne vallée de l'Euphrate (Cauvin 2002; Delerue 2007). Les datations radiocarbone provenant de Kalatepe ont permis de dater la diffusion initiale des technologies laminaires bipolaires dans la région à la phase de transition PPNB ancien-moyen (environ $8100 \mathrm{cal}$. BCE), notons que c'est à peu près au même moment que la diffusion de l'obsidienne vers le Levant se développe (Binder 2002; Binder \& Balkan-Atl1 2001).

D’une manière générale, l'organisation de la séquence de débitage bipolaire à Kalatepe s'inscrit dans la définition des modalités d'extraction les plus répandues de lames prédéterminées en superposition documentées dans le Levant nord (Abbès 2003) et du schéma " predetermined-upsilon » du Levant sud (Barzilai 2013). Cependant, la production de lames bipolaires en obsidienne de Kalatepe délivre des caractéristiques tout à fait distinctives, très rarement observées sur les nucléus en silex du Levant (Mureybet phase IVB et de Slenfe), celles-ci comprennent : 1) une forme de nucléus allongée avec des surfaces de débitage très étroites d'environ $3 \mathrm{~cm}$ d'épaisseur, 2) une utilisation très stricte des plans de frappes opposés qui permet un niveau de standardisation très élevé dans les productions (même les nucléus rejetés reflètent cette standardisation !) et 3) une très faible productivité (Binder \& BalkanAtlı 2001) (Figure 12). Des nucléus naviformes semblables à ceux de Kalatepe ont aussi été découverts au sein d'un autre atelier multi-périodes à Kayırl1-Bitlikeler. Toutefois, les nucléus retrouvés dans cet atelier sont moins standardisés et montrent une certaine variabilité dans les modalités de préparation et de gestion des nucléus à crête postéro-latérale et de nucléus à dos non-modifié (Balc1 2013). A Asıklı, les études initiales sur les assemblages bipolaires ont conclu que la présence d'un schéma de débitage bipolaire particulier (pour une consommation locale) est orientée vers la production de lames pointues (appointées) en série juxtaposée (Abbès et al. 1999). Ce troisième schéma, en complément des deux autres fondés sur l'extraction de lames prédéterminées en superposition largement répandus dans le Levant nord, s'intègre aux systèmes bipolaires tels que défini par Abbès (2003). Toutefois ce schéma n’a pas été défini avec le même degré de précision que les schémas superposés, et doit faire l'objet d'une analyse plus approfondie à l'avenir.

Les recherches engagées dans la région du Golludağ depuis la dernière décennie ont fourni une abondance de données nouvelles. Une série d'ateliers de taille d'obsidienne datant 
du Néolithique et du Chalcolithique et divers abris sous roche ont été identifiés, corroborant une utilisation largement répandue de la TLB dans la région et élargissant leur cadre chronologique (Balkan-Atlı et al. 2013). Notons d'ailleurs que les nucléus de type « naviforme » standardisés de Kalatepe sont absents des sites préhistoriques découverts récemment ainsi que d'autres sites bien connus tels qu'Aşıklı, Can Hasan III, Musular et Çatal Hoÿük. De même les produits standardisés de ces nucléus «naviformes » sont rares dans les niveaux acéramiques d'Aşıklı. Ces produits ont d'ailleurs été interprétés comme des pièces importées, suggérant qu'Aşıklı n'était pas une place centrale assurant le contrôle et la distribution de l'obsidienne de Cappadoce pendant la première moitié du $8^{\text {ème }}$ millénaire cal. BCE (Balc1 2013; Binder \& Balkan-Atl1 2001). Les résultats confirment également que la matière première exclusive utilisée pour la production des lames bipolaires pendant la période acéramique en Anatolie centrale est une obsidienne translucide de couleur gris-clair. Seul le site de Musular a fourni quelques rares lames bipolaires en silex (Balkan-Atlı et al. 2001).
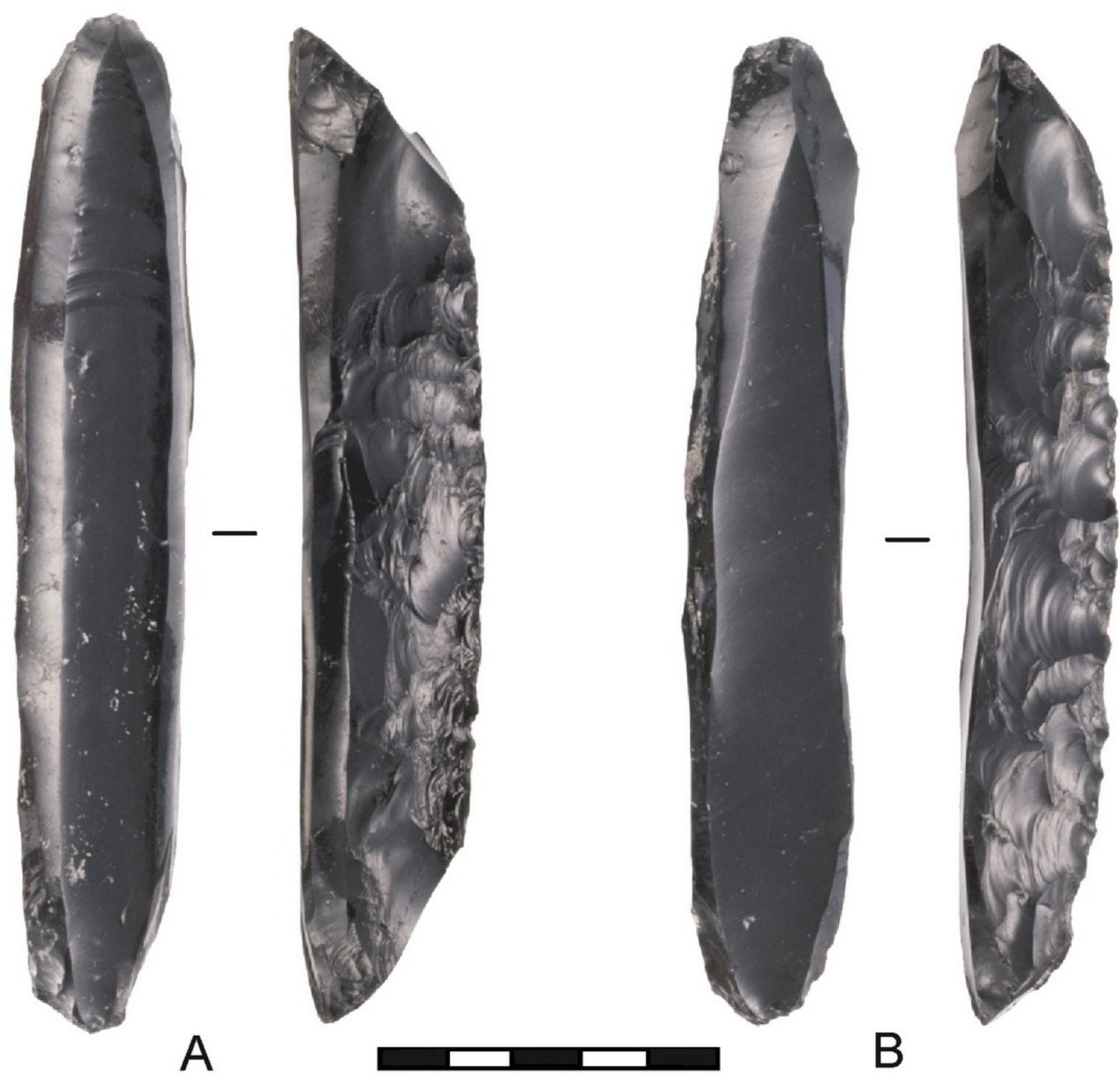

Figure 12. Nucléus naviforme de Kömürcü-Kaletepe (photo : Benoit Biraud, archive de Kömürcü-Kaletepe) (Balc1 2013). La barre d'échelle mesure $5 \mathrm{~cm}$ de long, avec des segments de $1 \mathrm{~cm}$.

Figure 12. Naviform cores from Kömürcü-Kaletepe (photo by Benoit Biraud, archive of Kömürcü-Kaletepe) (Balc1 2013). The scale bar is $5 \mathrm{~cm}$ long, with $1 \mathrm{~cm}$ segments. 
En dernier lieu, les technologies laminaires bipolaires sont encore utilisées durant le Néolithique céramique et même jusqu'au Chalcolithique dans quelques sites incluant Çatal Höyük et Tepecik Ciftlik. Cependant ces technologies bipolaires représentent une composante très minoritaire où les produits sont moins standardisés et la production se fait à l'extérieur des sites. Dans certains cas même leur présence pourrait résulter de réutilisation de matériaux de surface provenant d'autres ateliers PPNB (Balci 2013; Carter \& Milić 2013).

\section{Conclusions}

\subsection{Origines et signification}

La technologie laminaire bipolaire trouve son origine dans la moyenne vallée de l'Euphrate au cours de la fin du PPNA et des premiers stades du PPNB ancien (environ 8800 cal. BCE). L'apparition de cette production standardisée de grandes lames à caractéristiques très spécifiques (profils rectilignes, nervuration régulière, naturellement pointues et généralement robustes) pour la fabrication de pointes de projectiles, et dans une moindre mesure, de lames faucilles, de couteaux et d'autres types outils constitue un changement majeur dans les traditions lithiques locales. L'augmentation importante des dimensions des pointes de projectile liée à la technologie bipolaire, en comparaison des assemblages épipaléolithiques et PPNA de la région (dominés par les microlithes géométriques et des petites pointes de projectile comme les pointes d'El Khiam), instaure un changement remarquable dans la technologie des projectiles. Ce changement pourrait d'ailleurs être associé à un remplacement de l'arc par le propulseur dans la moyenne vallée de l'Euphrate (Borrell \& Štefanisko 2016).

Déterminer ce qui a conduit ces communautés à produire des quantités importantes de pointes de projectiles robustes et standardisées (qu'il s'agisse de pointes de flèches ou de javelines) reste encore à élucider. Un large éventail d'hypothèses a été proposé au cours des dernières décennies (développement d'une spécialisation artisanale de production lithique ; changement vers la chasse d'espèces plus grosses ; changement marqué dans l'organisation de la chasse; évolution de la perception sociale de la chasse ou encore augmentation de la concurrence intercommunautaire, conflit et guerres) (Abbès 2003; Bar-Yosef 2014; Nishiaki 2000; Quintero 2010) mais aucune n'est suffisamment concluante. Il est à noter que cette technologie laminaire bipolaire est traditionnellement associée à des sociétés pleinement néolithiques reposant sur la domestication achevée d'animaux et de plantes comme c'est le cas dans la majeure partie du Levant vers le milieu du $8^{\text {ème }}$ millénaire cal. BCE. Cependant, les contextes socio-économiques au sein desquels ces technologies ont émergées et se sont développées pour la première fois (PPNB ancien, environ $8800 \mathrm{cal}$. BCE) sont ceux de cultivateurs émergeants où la chasse est encore la principale source de protéines (Gourichon \& Helmer 2008; Helmer et al. 2005). Par conséquent, si ces technologies laminaires bipolaires ont émergé au sein de sociétés de chasseurs et non d'agriculteurs et d'éleveurs, il est donc raisonnable de supposer que leur apparition pourrait être liée à de grands changements dans les stratégies de chasse (Abbès 2003), mais la détermination de ces changements (techniques, organisation, valeur économique ou symbolique, proies, etc.) demeure encore problématique et nécessite des études plus approfondies.

\subsection{Est-ce une production spécialisée ?}

Sa vaste distribution spatiale dans des contextes socio-économiques variés (d'agriculteurs-éleveurs principalement mais aussi de chasseurs-cueilleurs) de même que l'utilisation de diverses matières premières (obsidienne et une grande variété de silex locaux et non-locaux) et la variabilité régionale croissante observée au sein du Levant suggèrent que 
la TLB n'a à priori, pas exigé l'existence d'une infrastructure économique soutenant une quelconque forme de spécialisation artisanale. Par conséquent, il apparaît nécessaire de repenser si, et dans quelles circonstances les technologies bipolaires peuvent être envisagées comme productions spécialisées. En guise d'exemple, il a été proposé, dans le sud du Levant, que des centres de production spécialisée ('Ain Ghazal et Yiftahel par exemple) ont exporté une partie de leur production vers d'autres localités (Milevski et al. 2008; Quintero 2010). Les principaux arguments d'une telle interprétation sont la quantité des productions (massive) ainsi que les niveaux élevés de savoir-faire et de standardisation atteints sur ces sites. En ce sens, il se pourrait bien que dans certaines circonstances, la TLB soit devenue une production spécialisée (à l'échelle d'un établissement, d'une maisonnée ou d'un individu) dans le but de créer un excédent, mais des preuves directes et concluantes d'un tel type d'échange et de la manière dont il fonctionne n’ont pas encore été signalées à ce jour. En outre, le nombre croissant de sites PPNB, allant de grands villages tels que Kharaysin (Ibáñez et al. 2015) à des sites cultuels ou mortuaires tels que Kfar HaHoresh (Barzilai \& Goring-Morris 2010), òu une production intensive et in situ de lames bipolaires est attesté, pose problèmes quant à l'identification des consommateurs potentiels de cette production spécialisée. Par exemple, qui consommait l'excédent d'un centre de production spécialisé tel que 'Ain Ghazzal si le grand village voisin de Kharaysin, un consommateur potentiel, produisait aussi intensivement des lames bipolaires?

\subsection{La diffusion : Ou ? Et comment ?}

Il est admis que la technologie laminaire bipolaire est originaire de la moyenne vallée de l'Euphrate et personne ne préconise pour l'instant d'autres centres d'innovations. De cette région centrale, elle s'est rapidement développée dans tout le Levant pour atteindre son

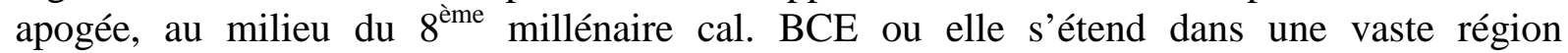
comprenant la vallée de l'Euphrate, les marges méridionales de la péninsule du Sinaï, Chypre et la Cappadoce. La nature d'une diffusion si rapide à travers un vaste cadre géographique constitue encore l'un des aspects les plus discutés et les plus complexes de la recherche néolithique au Levant. Le débat ne se limite pas à déterminer si il s'agit d'un transfert de technologie à grande distance où d'un processus démographique de "colonisation ». Le fait de connaitre d'autres éléments du répertoire culturel, si il en est, qui ont accompagné cette diffusion des technologies bipolaires est devenu partie intégrante du débat sur l'origine de la culture PPNB dans le sud du Levant (et la Cappadoce dans une moindre mesure). Au début des années 90, J. Cauvin (1994) préconisait clairement une expansion démographique à partir de la vallée de l'Euphrate vers le Levant sud. Alors que l'ampleur d'une telle « colonisation » pourrait faire l'objet d'un autre débat, de nombreux auteurs ont soutenus et agrémenté la primauté du Levant nord et le concept d'une zone nucléaire levantine (Edwards et al. 2004; Gopher et al. 2001; Kuijt 2003, par exemple). Cependant, au cours des dernières décennies, les découvertes récentes dans le Levant sud ont fourni des preuves croissantes d'une présence PPNB ancien dans la région. Ces nouvelles données permettent de proposer des interprétations alternatives en suggérant un développement local du PPNB dans la région et en supposant une interaction importante entre les deux régions (Ibáñez et al. 2010; Khalaily et al. 2007).

En Cappadoce, ou le débat a été moins intense au cours des dernières années, les différences observées dans la TLB dans la région (essentiellement entre Kalatepe et Aşıklı) ont été interprété comme résultant de deux traditions culturelles différentes (la culture d'Aşıklı et la culture PPNB Levantine) coexistant non loin des sources d'obsidienne entre 8000 et $7600 \mathrm{cal}$. BCE (Binder \& Balkan-Atl1 2001). Un tel argument suppose une expansion 
démographique du Levant nord vers l'Anatolie centrale, bien que l'ampleur de cette migration et la relation entre ces deux entités ne soit pas encore bien établie.

Le débat sur les conditions de la diffusion de la TLB est encore loin d'être clos et seul des approches régionales pluridisciplinaires et plus précises d'un point de vue chronologique permettront de fournir des éléments afin de mieux cerner les processus complexes (qu'ils soient culturels ou démographiques) engagés dans les développements du Néolithique de l'aile occidentale du Croissant fertile. Du point de vue des industries lithiques, l'expansion rapide à travers divers écosystèmes (déserts, fleuves, montagnes, steppe) ainsi que l'adoption de la TLB par des communautés fondées sur des modes de subsistance différents suggèrent que cette propagation est partie intégrante d'une diffusion culturelle majeure plutôt que le simple résultat de l'adoption d'une méthode de débitage qui offre certains avantages par rapport aux traditions lithiques antérieures. En ce sens, notre interprétation est, du moins en partie, en accord avec l'hypothèse de J. Cauvin (1994). Mais établir s'il s'agit d'une diffusion migratoire ou d'un transfert culturelle et définir quels autres éléments de la culture matérielle ont pu accompagner cette diffusion est au delà de la portée de cet article et du potentiel d'interprétation des analyses lithiques.

\subsection{D’une homogénéité durable à des développements culturels régionaux de plus en plus divergents}

Les technologies laminaires bipolaires constituent l'élément le plus caractéristique de la culture matérielle du PPNB indiquant une forte connexion culturelle entre le nord et le sud du Levant. Après plusieurs siècles d'homogénéité remarquable, un changement s'opère au sein des industries lithiques du Levant et les technologies bipolaires déclinent. Cet abandon s'initie dans diverses circonstances et à différentes époques en fonction des régions (environ 7200 cal. BCE dans le Levant nord et environ 6500-6400 cal. BCE dans le Levant sud) (Borrell \& Khalaily 2016). Un tel décalage chronologique dans l'abandon de l'élément le plus commun de la culture matérielle PPNB révèle des développements culturels divergents prenant place entre 7200 et 6900 cal. BCE (Borrell \& Khalaily 2016). Ce processus de «déconnexion » pourrait trouver ses racines au cours des siècles précédents, comme l’indique le fait que la stratégie bipolaire " off-set ", caractéristique du PPNB moyen et récent du Levant nord entre 7800-7100 cal. BCE, n’a jamais diffusé vers le sud du Levant, ce qui pourrait signifier que les éléments ayant permis et facilité la diffusion initiale de la TLB au Levant sud ne fonctionnaient plus à cette période.

Les causes de l'abandon de la TLB ont également fait l'objet d'études approfondies au cours des dernières décennies. Diverses hypothèses ont été proposées comme la diminution des activités de chasse (Abbès 2003) en conjonction avec l'intensification des pratiques agricoles (Nishiaki 2000), ou l'effondrement de l'infrastructure économique régionale soutenant la spécialisation artisanale, cette dernière hypothèse étant proposée par les auteurs considérant les technologies laminaires bipolaires en tant que productions spécialisées (Quintero 2010). Ces diverses hypothèses pourraient être valables pour certaines régions, mais s'appliquent difficilement à l'ensemble du Levant, ce qui suggère que les causes de l'abandon de l'élément le plus commun de la culture matérielle du PPNB sont diverses ; aussi diverses que les chronologies et les contextes socio-économiques au sein desquels les TLB se développent dans tout le Levant (Borrell \& Khalaily 2016).

\section{Remerciments}

Je tiens à exprimer ma gratitude à $\mathrm{F}$. Abbès, S. Balc1, O. Barzilai, D. Binder, J.J. Ibáñez et $\mathrm{H}$. Khalaily pour m'avoir permis d'utiliser et de modifier les dessins, images et schémas qu'ils ont produits et publiés. F. Abbès, O. Barzilai et E. Healey ont également contribué aux 
versions antérieures de cet article par leurs commentaires précieux. Les commentaires et les critiques de deux examinateurs anonymes ont également été d'une grande valeur.

\section{Références}

Abbès, F. 1998, Réflexions concernant les nucleus bipolaires et naviformes du Proche-Orient Néolithique. Cahiers de l'Euphrate, 8: 139-150. (in French) ("Reflections on bipolar and naviform cores of the Neolithic Near East”)

Abbès, F., \& Déraprahamian G. 2002, Pression et Percussion: Identification des stigmates sur des nucléus naviformes (Syrie), In: Préhistoire et approche expérimentale

(Bourguignon, L., \& Ortega, L., Eds.), Série Préhistoire Vol. 5, Editions M. Mergoal, Montagnac: p. 195-205. (in French) ("Pressure and Percussion: Identification of stigmas on naviform cores”)

Abbès, F. 2003, Les outillages néolithiques en Syrie du Nord. Méthode de débitage et gestion laminaire durant le PPNB. BAR International Series Vol. 1150, Archaeopress, Oxford, 235 p. (in French) ("Neolithic tool kit in Northern Syria. Method of knapping and blade management during the PPNB")

Abbès, F. 2007, Les débitages laminaires de la fin du PPNA (Jerf el Ahmar, Mureybet, Cheikh Hassan). In: Systèmes techniques et communautés du néolithique précéramique au Proche-Orient (Astruc, L., Binder, D., \& Briois, F., Eds.), Éditions APDCA, Antibes: p. 127-136. (in French) ("Blade production at the end of the PPNA (Jerf el Ahmar, Mureybet, Cheikh Hassan)”)

Abbès, F. 2008, Analyse technologique, In: Le site néolithique de Tell Mureybet (Syrie du Nord) (Ibáñez, J.J, Ed.), BAR International Series Vol. S1843, Archaeopress, Oxford: p. 237-280. (in French) (“The Neolithic site of Mureybet (Northern Syria)")

Abbès, F., Balkan-Atlı, N., Binder, D., \& Cauvin, M.C. 1999, Étude préliminaire de l'industrie lithique d'Asıklı Höyük. Turkish Academy of Sciences Journal of Archaeology, Tüba Ar, 2: 117-137.(in French) ("Preliminary study of the lithic industries of Asıklı Höyük”)

Akazawa, T. 1979, Flint Factory Sites in Palmyra Basin. In: Paleolithic sites of Douara Cave and Paleogeography of Palmyra Basin in Syria (Hanihara, K., \& Akazawa, T., Eds.), The University Museum Bulletin Vol. 16. University of Tokyo Press, Tokyo: p. 159200. (in English) (“Ateliers de taille de silex dans le bassin de Palmyre”)

Arimura, M. 2007, Néolithisation de la Syrie du Nord-Ouest. L'evolution des industries lithiques à Tell Ain el-Kerkh (bassin du Rouj, 8500-600 cal. BC). Unpublished PhD thesis. Université Lyon 2, Lyon, 606 p. (in French) ("The neolithisation of north-west Syria. The evolution of the lithic industries of Tell Ain el-Kerkh")

Aurenche, O., Cauvin, J., Cauvin, M.-C., Copeland, I., Hours, F., \& Sanlaville P. 1981, Chronologie et organisation de l'espace dans le Proche-Orient de 12000 à 5600 avant J.-C. In: Préhistoire du Levant (Cauvin, J., \& Sanlaville, P., Eds.), CNRS Éditions, Paris: p. 571-601. (in French) ("Chronology and organization of space in the Near East from 12000 to 5000 BC”)

Baird, D. 1994, Chipped Stone Production Technology from the Azraq Project Neolithic Sites. In: Neolithic Chipped Stone Industries of the Fertile Crescent (Gebel, H.G.K., \& 
Kozłowski, S.K., Eds.), SENEPSE Vol. 1, Ex Oriente, Berlin: p. 525-541. (in English) (“Technologie de la pierre taillée des sites néolithiques du projet Azraq")

Balc1, S. 2013, Naviform technology at Golludağ, Central Anatolia: Some remarks. In: Stone tools in transition. From Hunter-Gatherers to Farming Societies in the Levant (Borrell, F., Ibáñez, J.J., \& Molist, M., Eds.), Universitat Autònoma de Barcelona, Bellaterra: p. 277-289. (in English) (“Technologie naviforme à Golludağ, Anatolie centrale: quelques remarques")

Balkan-Atlı, N. 1994, The Typological Characteristics of the Aşıklı Höyük Chipped Stone Industry. In: Neolithic Chipped Stone Industries of the Fertile Crescent (Gebel, H.G.K., \& Kozłowski, S.K., Eds.), SENEPSE Vol. 1, Ex Oriente, Berlin: p. 209-222. (in English) ("Les caractéristiques typologiques des industries lithiques taillées d'Aşıklı Höyük”)

Balkan-Atlı, N., Cauvin, M.C., Der Aprahamian, G., \& Kuzucuoğlu, C. 1997, Rapport sur l'obsidienne cappadocienne et sa diffusion, campagne 1996. Anatolia Antiqua, 5: 263274. (in French) ("Report about Cappadocian obsidian and its diffusion, season 1996") URL: http://www.persee.fr/doc/anata_1018-1946_1997_num_5_1_879

Balkan-Atlı, N., Binder, D., \& Kuzucuoğlu, C. 1999, L'atelier néolithique de KömürcüKalatepe: fouilles de 1998. Anatolia Antiqua, 7: 231-243. (in French) ("The Neolithic workshop of Kömürcü-Kalatepe: 1998 excavations”) URL: www.persee.fr/doc/anata_1018-1946_1999_num_7_1_929

Balkan-Atlı, N., Kayacan, N., Özbaşaran, M., \& Yıldırım. 2001, Variability in the Neolithic arrowheads of Central Anatolia (typological, technological and chronological aspects). In: Beyond tools (Caneva, I., Lemorini, C., Zampetti, D., \& Biagi, P., Eds.), SENEPSE Vol. 9, Ex Oriente, Berlin: p. 27-43. (in English) ("Variabilité des pointes de flèche néolithiques de l’Anatolie centrale (aspects typologiques, technologiques et chronologiques")

Balkan-Atl1, N., Kayacan, N., Balc1, S. Astruc, L., \& Erturaç, K. 2013, Göllü Dağ Obsidian Project. In: Stone tools in transition. From Hunter-Gatherers to Farming Societies in the Levant (Borrell, F., Ibáñez, J.J., \& Molist, M., Eds.), Universitat Autònoma de Barcelona, Bellaterra: p. 465-474. (in English) (“Projet obsidienne de Göllü Dağg”)

Bar-Yosef, O. 1981, Neolithic Sites in Sinai. In: Beitage zur Umweltgeschichte des Vorderen Orients (Frey, W., \& Uerpmann, H.P., Eds.), Beihefte zum Tuebinger Atlas des Vorderen Orients Reihe A8, Ludwig Reichert, Weisbaden: p. 217-235. (in English) ("Sites néolithiques au Sinaï”)

Bar-Yosef, O. 2014, The homelands of the Cyprus colonizers. Eurasian Prehistory, 10(1-2): 67-82. (in English) ("La patrie des colonisateurs de Chipre”)

URL: https://www.peabody.harvard.edu/files/5_O.Bar-Yosef_start.pdf

Barzilai, O. 2010, Social Complexity in the Southern Levantine PPNB as Reflected through Lithic Studies. BAR International Series Vol. 2180, Archaeopress, Oxford, 194 p. (in English) (“Complexité sociale au Levant sud a partir des études lithiques”)

Barzilai, O. 2013, The bidirectional blade industries of the southern Levant. In: Stone tools in transition. From Hunter-Gatherers to Farming Societies in the Levant (Borrell, F., Ibáñez, J.J., \& Molist, M., Eds.), Universitat Autònoma de Barcelona, Bellaterra: p. 5972. (in English) (“Les industries laminaires bipolaires du Levant sud”) 
Barzilai, O., \& Garfinkel, Y. 2006, Bidirectional Blade Technology after the PPNB: New Evidence from Sha’ar Hagolan, Israel. Neo-Lithics, 1(6): 27-31. (in English) (“La technologie laminaire bipolaire après le PPNB : nouvelles évidences de Sha'ar Hagolan”) URL: http://www.exoriente.org/docs/00065.pdf

Barzilai, O., \& Goring-Morris, A.N. 2010, Bidirectional Blade Production at the PPNB Site of Kfar Ha-Horesh: The Techno-Typological Analysis of a Workshop Dump. Paléorient, 36(2): 5-34. (in English) ("Production laminaire bipolaire au site PPNB de Kfar HaHoresh”) doi:10.3406/paleo.2010.5386

Barzilai, O., \& Goring-Morris, A.N. 2011, Nahal Lavan 1021, A PPNB Workshop Site in the Western Negev Dunes. In: The state of the stone terminologies, continuities and contexts in Near Eastern lithics (Healey E., Campbell S., \& Maeda, O., Eds.), SENEPSE Vol. 13, Ex Oriente, Berlin: p. 65-74. (in English) ("Nahal Lavan 1021, un atelier PPNB dans les dunes du Néguev occidental”)

Barzilai O., \& Goring-Morris A.N. 2013, An estimator for bidirectional (naviform) blade productivity in the Near Eastern Pre-Pottery Neolithic B. Journal of Archaeological Science, 40(1): 140-147. (in English) (“Un estimateur de la productivité de lames bipolaires (naviforme) dans le Néolithique précéramique B du Proche-Orient”) doi:10.1016/j.jas.2012.06.025

Binder, D. 2002, Stones making sense: what obsidian could tell about the origins of the Central Anatolian Neolithic. In: The Neolithic of Central Anatolia (Gérard, F., \& Thissen, L., Eds.), EGE Yayınları, Istanbul: p. 79-90. (in English) ("Pierres faisant sens : ce que l'obsidienne pourrait dire sur les origines du Néolithique d'Anatolie central")

Binder, D. 2008, Technologie lithique et comportement social dans le PPN de Çayönü tepesi (Turquie). Paléorient, 34(1): 5-21. (in French) ("Lithic technology and social behaviour in PPN Çayönü (Turkey)”) doi:10.3406/paleo.2008.5230

Binder, D. \& Balkan-Atl1, N. 2001, Obsidian and blade technology at Komurcu-Kaletepe (Central Anatolia). In: Beyond tools (Caneva, I., Lemorini, C., Zampetti, D., \& Biagi, P., Eds.), SENEPSE Vol. 9, Ex Oriente, Berlin: p. 1-16. (in English) (“Obsidienne et technologie laminaire à Kumurcu-Kalatepe (Anatolie central)”)

Borrell, F. 2011a, Changes in chipped stone industries in south-eastern Anatolia: Akarcay Tepe (7,600-6,800 cal. BC). In: The state of the stone terminologies, continuities and contexts in Near Eastern lithics (Healey E., Campbell S., \& Maeda, O., Eds.), SENEPSE Vol. 13, Ex Oriente, Berlin: p. 213-226. (in English) ("Changements dans les industries lithiques dans les sud-est de l’Anatolie: Akarçay Tepe (7,600-6,800 cal. $\mathrm{BC})$ )

Borrell, F. 2011b, Knapping methods and techniques at Tell Halula (middle Euphrates valley), during the mid VIII ${ }^{\text {th }}$ millennium cal. BC. In: The state of the stone terminologies, continuities and contexts in Near Eastern lithics (Healey E., Campbell S., \& Maeda, O., Eds.), SENEPSE Vol. 13, Ex Oriente, Berlin: p. 291-303. (in English) ("Méthodes et techniques de taille à Tell Halula (vallée moyenne de l'Euphrate), au milieu du VIII ${ }^{\text {ème }}$ millénaire avant JC”)

Borrell, F. 2011c, Bi-directional Blade Technology in the Northern Levant During the 7-8 ${ }^{\text {th }}$ Millennia CAL B.C: New Insights from Mamarrul Nasr 2, Syria. Journal of Field Archaeology, 36(2): 132-150. (in English) (“Technologie laminaire bipolaire dans les 
Levant nord durant le 7-8 ${ }^{\text {ème }}$ millénaire avant JC : Nouvelles perspectives de Mamarrul Nasr 2, Syrie”) doi:10.1179/009346911x12991472411240

Borrell, F. 2015, Further remarks about lithic production at Akarçay Tepe (middle Euphrates valley) during the Late PPNB. In: Broadening Horizons 4: A Conference of Young Researchers Working in the Ancient Near East, Egypt and Central Asia (Affanni, G., Baccarin, C., Cordera, L., di Michele, A. \& Gavagnin, K. Eds.), Bar International Series 2698, Archaeopress, Oxford: p. 265-277. (in English) ("Remarques sur la production lithique à Akarçay Tepe (moyenne vallée de l'Euphrate) durant le PPNB récent”)

Borrell, F., \& Molist, M. 2014, Social interaction at the end of the Pre-Pottery Neolithic B: An inter-site analysis in the Euphrates valley. Cambridge Archaeological Journal, 24(2): 215-232. (in English) (“Interaction sociale à la fin du Néolithique précéramique B : Une analyse inter-sites dans la vallée de l’Euphrate”) doi:10.1017/S0959774314000456

Borrell, F., \& Khalaily, H. 2016. Reconstructing the Last Stages of Bidirectional Blade Technology in the Levant: North and South (dis)connections. Paléorient, 42(2): 73-95. (in English) ("Reconstruction des dernieres étapes de la technologie laminaire bipolaire dans le Levant : (des)connexions nord et sud ”)

Borrell, F., \& Štefanisko, D. 2016, Reconstructing projectile technology during the PrePottery Neolithic B in the Levant: A multi-variant approach to large tanged points from Halula. Journal of Archaeological Science, 69: 130-142. (in English) ("Reconstruction de la technologie des projectiles pendant le Néolithique précéramique B au Levant : une approche multi-variant des grandes pointes à pédoncule”) doi:10.1016/j.jas.2016.04.005

Borrell, F., Boëda, E., Molist, M., al-Shakel, H., \& Vicente, O. 2011, New evidence concerning the Neolithisation process of the central Syrian desert. The Neolithic complex of Mamarrul Nasr. Paléorient, 37(2): 35-46. (in English) ("Nouvelles données concernant le processus de néolithisation du désert syrien central. Le complexe néolithique de Mamarrul Nasr”) doi:10.3406/paleo.2011.5421

Borrell, F., Boëda, E., Molist, M., \& al-Shakel, H. 2013, The first half of the 7th millennium cal. BCE in the El Kowm basin, central Syria: Umm el-Tlel revisited. In: Interpreting the Late Neolithic of Upper Mesopotamia (Nieuwenhuyse, O.P., Akkermans P.M.M.G., Bernbeck, R., \& Rogasch, J., Eds.), PALMA Series Vol. 9, Brepols, Turnhout: p. 277287. (in English) ("La première moitié du $7^{\text {ème }}$ millénaire cal. BCE dans le bassin d'El Kowm, Syrie centrale : Umm el-Tlel revisité ”)

Briois, F., Vigne, J.D., \& Guilaine, J. 2013, The early cypriot Pre-Pottery Neolithic: New evidence from Amathus area. In: Stone tools in transition. From Hunter-Gatherers to Farming Societies in the Levant (Borrell, F., Ibáñez, J.J., \& Molist, M., Eds.), Universitat Autònoma de Barcelona, Bellaterra: p. 299-313. (in English) ("Le début du Néolithique précéramique chypriote : nouvelles données de la région d’Amathus”)

Calley, S. 1986a, Technologie du débitage à Mureybet, Syrie: 9e-8e millénaires, BAR International Series Vol. 312. Archaeopress, Oxford-Lyon, 438 p. (in French) ("Debitage technology in Mureybet, Syria: $9^{\text {th }}-8^{\text {th }}$ millennia")

Calley S. 1986b, L’atelier de Qdeir 1 en Syrie : exploitation des nucléus naviformes à la fin du PPNB, 6e millénaire. Première approche. Paléorient, 12(2): 49-67. (in French) (“The Qdeir 1 workshop in Syria: Exploitation of naviform cores at the end of the PPNB, $6^{\text {th }}$ millennium. First approach”) doi:10.3406/paleo.1986.4408 
Calley, S. 1988, Some questions concerning ‘upsilon’ blades. Anatolica, 15: 87-92. (in English) (“Quelques questions concernant les lames 'upsilon’”)

Caneva, I., Iovino, M.R., Lemorini, C., Ozdogan, A., \& Zampetti, D. 2001, A combined analysis of lithic assemblages from Çayönü. In: Beyond tools (Caneva, I., Lemorini, C., Zampetti, D., \& Biagi, P., Eds.), SENEPSE Vol. 9, Ex Oriente, Berlin: p. 165-181. (in English) (“Une analyse combinée des assemblages lithiques de Çayönü”)

Caracuta, V., Weiss, E., van den Brink, E., Liran, R., Vardi, J., \& Barzilai, O. 2014, From natural environment to human landscape: New archaeobotanical data from the Neolithic site of Nahal Zippori 3, Lower Galilee. Neo-lithics, 1(14): 33-41. (in English) ("De l'environnement naturel au paysage humain : nouvelles donées archéobothaniques du site néolithique de Nahal Zippori 3, basse Galilée”)

Carter, T., \& Milić, M. 2013, The consumption of obsidian at Neolithic Çatalhöyük: A longterm perspective. In: Stone tools in transition. From Hunter-Gatherers to Farming Societies in the Levant (Borrell, F., Ibáñez, J.J., \& Molist, M., Eds.), Universitat Autònoma de Barcelona, Bellaterra: p. 495-508. (in English) ("La consummation d’obsidienne au néolithique Çatalhöyük : une perspective à long terme”)

Cauvin, J. 1968, Les Outillages Néolithiques de Byblos et du Littoral Libanais. Fouilles de Byblos, tome IV. Adrien Maisonneuve, Paris, 360 p. (in French) ("The Neolithic Tools of Byblos and the Lebanese Coast. Byblos excavations”)

Cauvin, J. 1989, La stratigraphie de Cafer Höyük Est (Turquie) et les origines du PPNB du Taurus. Paléorient, 15(1): 75-86. (in French) ("The stratigraphy of Cafer Höyük East (Turkey) and the origins of the PPNB of the Taurus”) doi:10.3406/paleo.1989.4486

Cauvin, J. 1994, Naissance des divinités, naissance de l'agriculture : la révolution des symboles au Néolithique. Editions du CNRS, Paris, 304 p. (in French) ("Birth of divinities, birth of agriculture: The symbols revolution in the Neolithic”)

Cauvin, M.-C. 1994, Synthèse sur les industries lithiques Néolithique Précéramique en Syrie. In: Neolithic Chipped Stone Industries of the Fertile Crescent (Gebel, H.G.K., \& Kozłowski, S.K., Eds.), SENEPSE Vol. 1, Ex Oriente, Berlin: p. 279-298. (in French) ("Synthesis on the Pre-Pottery Neolithic lithic industries in Syria”)

Cauvin, M.-C. 2002, L'obsidienne et sa diffusion dans le Proche-Orient Néolithique. In: Matériaux, Productions, Circulations du Néolithique à l'Age du Bronze (Guilaine, J., Ed.), Séminaire du Collège de France, Collection des Hesperides Editions Errance, Paris: p. 13-30. (in French) ("Obsidian and its diffusion in the Neolithic Near East")

Cauvin, M.-C., \& Cauvin, J. 1993, La Séquence Néolithique PPNB au Levant Nord. Paléorient, 19(1): 23-28. (in French) (“The PPNB sequence in the Northern Levant”) doi:10.3406/paleo.1993.4579

Coqueugniot, E. 1994, L’industrie lithique de Dja’ de el Mughara et le début du PPNB sur l'Euphrate Syrien (Sondages 1991 et 1992). In: Neolithic Chipped Stone Industries of the Fertile Crescent (Gebel, H.G.K., \& Kozłowski, S.K., Eds.), SENEPSE Vol. 1, Ex Oriente, Berlin: p. 313-330. (in French) (“The lithic industry of Dja'de el Mughara and the beginning of the PPNB on the Syrian Euphrates (1991 and 1992 surveys)”)

Crowfoot-Payne, J. 1984, The flint industries of Jericho. In: Excavations at Jericho, V (Kenyon, K.M., \& Holland, T.A., Eds.), British School of Archaeology in Jerusalem, London: 622-659. (in English) (“Les industries en silex de Jéricho”) 
Delerue, S. 2007, L'obsidienne dans le processus de Neolithisation du Proche Orient (120006500 av. J.-C. cal.). Ph.D. dissertation, Université Bordeaux Montagne, Bordeaux, 541 p. (in French) ("Obsidian in the process of Neolithisation of the Near East (120006500 cal. BC”)

Dietrich, O. 2016, How old is it? Dating Göbekli Tepe.

URL: https://tepetelegrams.wordpress.com/ (last accessed August 2016). (in English) (“Quel âge a-t-il? Datant Göbekli Tepe”)

Edwards, P.C., Meadows, J., Sayej, G., and Westaway, M. 2004, From the PPNA to the PPNB: new views from the Southern Levant after excavations at Zahrat adh Dhra' 2 in Jordan. Paléorient, 30(2): 21-60. (in English) (“Du PPNA au PPNB : nouvelles vues du Levant sud après des fouilles à Zahrat adh Dhara' en Jordanie”) doi:10.3406/paleo.2004.1010

Eisenberg, E., Gopher, A., \& Greenberg, R. (Eds.) 2001, Tel Te'o. A Neolithic, Chalcolithic, and Early Bronze Age Site in the Hula Valley, IAA Report Series Vol. 13. Israel Antiquities Authority, Tel Aviv, 227 p. (in English) (“Tel Te’o. Un site néolithique, chalcolithique et de l'âge du Bronze ancien dans la vallée de Hula”)

Garfinkel, Y., Dag, D., Khalayli, H., Marder, O., Milevski, I., \& Ronen, A. 2012, The PrePottery Neolithic B Village of Yiftahel. The 1980s and 1990s Excavations. Bibliotheca neolithica Asia meridionalis et occidentalis. Ex-Oriente, Berlin, 310 p. (in English) ("Le village Néolithique précéramique B de Yiftahel. Les fouilles des années 1980 et 1990”)

Gebel, H.G.K. 2004, Central to what? The centrality issue of the late PPNB megasite phenomenon in Jordan. In: Central settlements in neolithic Jordan (Bienert, H.D., Gebel, H.G.K., \& Neef, R., Eds.), SENEPSE Vol. 5, Ex Oriente, Berlin: p. 1-19. (in English) ("Central à quoi? La question centrale du phénomene des mégasites du PPNB recent en Jordanie”)

Getzov, N. 1999, Ha-Gosherim. Hadashot Arkheologiyot (Excavations and Surveys in Israel), 110: 2-3. (in English) (“Ha-Gosherim”)

Gopher, A. 1989, The flint assemblages of Munhatta - final report, Les Cahiers du CRFJ, Vol. 4). Association Paléorient, Paris, 162 p. (in English) ("Les assemblages en silex de Munhatta - rapport final”)

Gopher, A. 1997, Horvat Galil - An Early PPNB Site in the Upper Galilee, Israel. Tel Aviv, 24(2): 183-222. (in English) ("Horvat Galil - Un site du PPNB ancient en haute Galilée, Israël”) doi:10.1179/tav.1997.1997.2.183

Gopher, A., Abbo, S., \& Lev-Yadun, S. 2001, The "when”, the "where” and the "why” of the Neolithic revolution in the Levant. Documenta Praehistorica, 28: 49-62. (in English) ("Le “quand", le "où” et le "pourquoi” de la révolution néolithique au Levant") doi:10.4312/dp.28.3

Goring-Morris, N., Hovers, E., \& Belfer-Cohen A. 2009, The Dynamics of Pleistocene and Early Holocene Settlement Patterns and Human Adaptations in the Levant: An Overview. In: Transitions in Prehistory. Essays in Honour of Ofer Bar-Yosef (Shea J., \& Lieberman D., Eds.), Oxbow Books, Oxford and Oakville: p. 185-252. (in English) ("La dynamique des schémas de peuplement du Pléistocene et l'Holocène initial et les adaptations humaines au Levant : un aperçu”)

Gourichon, L., \& Helmer, D. 2008, Etude archéozoologique de Mureybet. In: Le site néolithique de Tell Mureybet (Syrie du Nord). En hommage à Jacques Cauvin (Ibáñez, 
J.J., Ed.), Maison de l’Orient et de la Méditerranée, BAR International Series Vol. 1843, Archeopress, Oxford and Lyon: p. 115-227. (in French) (“Archaeozoological study of Mureybet”)

Helmer, D., Gourichon, L., Monchot, H., Peters, J., \& Saña, M. 2005, Identifying early domestic cattle from Pre-Pottery Neolithic sites on the Middle Euphrates using sexual dimorphism. In: The First Steps of Animal Domestication: New Archaeozological Approaches (Vigne, J.-D., Peters, J., \& Helmer, D., Eds.), Oxbow Books, Oxford: p. 8695. (in English) ("Identifier les bovins domestiques des sites du Néolithique précéramique du moyen Euphrate en utilisant le dimorphisme sexuel”)

Ibáñez, J.J., Balbo, A., Braemer, F., Gourichon, L., Iriarte, E., Santana, J., \& Zapata, L. 2010, The early PPNB levels of Tell Qarassa North (Sweida, southern Syria). Antiquity: Project Gallery, 84(325). (in English) ("Les niveaux du PPNB ancient de Tell Qarassa (Sweida, sud de la Syrie)”) URL: http://www.antiquity.ac.uk/projgall/ibanez325/

Ibáñez, J.J., Muñiz, J., Iriarte, E., Monik, M., Santana, J., Teira, L., Corrada, M., Lagüera, M.A., Lendakova, Z., Regalado, E., \& Rosillo, R. 2015, Kharaysin: A PPNA and PPNB Site by the Zarqa River. 2014 and 2015 Field Seasons. Neo-Lithics, 2(15): 11-19. (in English) ("Kharaysin : un site PPNA et PPNB près de la rivière Zarqa. Campagnes 2014 et 2015”)

Khalaily, H., Bar-Yosef O., Barzilai, O., Boaretto, E., Bocquentin, F., Eirikh-Rose, A., Greenhut, Z., Goring-Morris, N., Le Dosseur, G., Marder, O., Sapir-Hen, L., \& Yizhaq, M. 2007, Excavations at Motza in the Judean Hills and the Early Pre-Pottery Neolithic B in the Southern Levant. Paléorient, 33(2): 5-37. (in English) ("Fouilles à Motza dans les collines de Judée et le début du Néolithique précéramique B ancien au Levant sud”) doi:10.3406/paleo.2007.5218

Kozłowski, S.K. 1999, The Eastern Wing of the Fertile Crescent. Later Prehistory of Greater Mesopotamian Lithic Industries. BAR International Series Vol. 760. Archaeopress, Oxford, 194 p. (in English) (“L’aile east du Croissant fertile. Préhistoire récent des grandes industries lithiques mésopotamiennes”)

Kozłowski, S.K., \& Aurenche, O. 2005, Territories, Boundaries and Cultures in the Neolithic Near East. BAR International Series Vol. 1362. Archaeopress, Oxford, 275 p. (in English) ("Territoires, frontières et cultures au Proche-Orient néolithique”)

Kuijt, I. 2003, Between foraging and farming: Critically evaluating the archaeological evidence for the southern Levantine Early Pre-Pottery Neolithic B Period. Turkish Academy of Sciences - Journal of Archaeology, 6: 7-25. (in English) (“Entre la chasse et la cueillette et l'agriculture : évaluation critique des indices archéologiques de la période Néolithique précéramique B”)

Lechevallier, M., \& Ronen, A. 1994, L'industrie lithique. In: Le gisement de Hatoula en Judée occidentale, Israël (Lechevallier, M., \& Ronen, A., Eds.), Association Paléorient, Paris: p. 141-180. (in French) (“The lithic industry”)

Matskevich, Z. 2011, The lithic assemblages of Sha'ar Hagolan: PPN/PN continuity? In: The state of the stone terminologies, continuities and contexts in Near Eastern lithics (Healey E., Campbell S., \& Maeda, O., Eds.), SENEPSE Vol. 13, Ex Oriente, Berlin: p. 227-241. (in English) (“Les assemblages lithiques de Sha'ar Hagolan : continuité $\mathrm{PPN} / \mathrm{PN}$ ?”) 
Milevski, I., Khalaily, H., Getzov, I., \& Hershkovitz, I. 2008, The Plastered Skulls and Other PPNB Finds from Yiftahel, Lower Galilee (Israel). Paléorient, 34(2): 37-46. (in English) ("Les crânes surmodelés en plâtre et autres trouvailles du PPNB de Yiftahel, basse Galilée (Israël)”) doi:10.3406/paleo.2008.5255

Mitki, N., Barzilai, O., \& Goring-Morris, N. 2013, Observations on the chaîne opératoire of bidirectional blade production at Nahal Lavan 1021 base on refitting studies. In: Stone tools in transition. From Hunter-Gatherers to Farming Societies in the Levant (Borrell, F., Ibáñez, J.J., \& Molist, M., Eds.), Universitat Autònoma de Barcelona, Bellaterra: p. 115-132. (in English) (“Observations sur la chaîne opératoire de la production de lames bipolaires à Nahal Lavan 1021 à partir d'études de remontages lithiques”)

Nadel, D., \& Nadler-Uziel, M. 2011, Is the PPNC really different? The flint assemblages from three layers at Tel Roim West, Hula Basin. In: The state of the stone terminologies, continuities and contexts in Near Eastern lithics (Healey E., Campbell S., \& Maeda, O., Eds.), SENEPSE Vol. 13, Ex Oriente, Berlin: p. 243-255. (in English) (“Le PPNC est-il vraiment different? Les assemblages de silex de trois couches à Tel Roim West, bassin de Hula")

Nishiaki, Y. 1994, The Naviform Method at Douara Cave II, Palmyra, Syria. In: Neolithic Chipped Stone Industries of the Fertile Crescent (Gebel, H.G.K., \& Kozłowski, S.K., Eds.), SENEPSE Vol. 1, Ex Oriente, Berlin: p. 363-378. (in English) (“La méthode naviforme à Douara Cave II, Palmyre, Syrie”)

Nishiaki, Y. 2000, Lithic Technology of Neolithic Syria. BAR International Series 840. Archaeopress, Oxford, 241 p. (in English) ("La technologie lithique de la Syrie néolithique")

Nishiaki, Y., \& Le Mière, M. 2005, The oldest pottery Neolithic of Upper Mesopotamia: New evidence from Tell Seker al-Aheimar, the Khabur, northeast Syria. Paléorient, 31(2): 55-68. (in English) ("La plus ancienne poterie néolithique de Haute Mésopotamie : nouvelle évidence de Tell Seker al-Aheimar, le Khabour, nord-est de la Syrie”) doi:10.3406/paleo.2005.5125

Perrot, J. 1968, La Préhistoire Palestinienne. Supplément au Dictionnaire de la Bible VIII, Letouzey et Ané, Paris: p. 286-446. (in French) ("Palestinian Prehistory”)

Quintero, L. 2010, Evolution of Lithic Economies in the Levantine Neolithic. Development and Demise of Naviform Core Technology as Seen at 'Ain Ghazal ('Ain Ghazal Excavation Reports, Volume 2). Bibliotheca neolithica Asiae meridionalis et occidentalis, Ex Oriente, Berlin, 184 p. (in English) (“Evolution des économies lithiques dans le Néolithique levantin. Développement et disparition de la technologie naviforme vue à 'Ain Ghazal”)

Quintero, L.A., \& Wilke, P.J. 1995, Evolution and Economic Significance of Naviform Coreand-Blade Technology in the Southern Levant. Paléorient, 21(1): 17-33. (in English) ("Évolution et importance économique de la technologie naviforme dans le Levant sud”) doi:10.3406/paleo.1995.4607

Rokitta-Kumnow, D. 2011, The Lithic Artifacts from the Late Neolithic Settlement of Shir/Western Syria. Zeitschrift für Orient-Archäologie. Band 4: 212-244. (in English) ("Les artefacts lithiques du site Néolithique tardif de Shir/Syrie occidentale")

Rollefson, G.O. 1990, Neolithic Chipped Stone Technology at 'Ain Ghazal, Jordan: The Status of the PPNC Phase. Paléorient, 16(1): 119-124. (in English) ("La technologie des 
pierres taillées néolithiques à 'Ain Ghazal, Jordanie : l'état de la phase PPNC”) doi:10.3406/paleo.1990.4526

Rollefson, G.O. 2001, The Neolithic Period. In: The Archaeology of Jordan (MacDonald, B., Adams, R., \& Bienkowski, P., Eds.), Sheffield Academic Press, Sheffield: p. 67-105. (in English) ("La période Néolithique”)

Rollefson, G.O., Simmons, A.H., Kafafi, Z. 1992. Neolithic Cultures at Áin Ghazal, Jordan. Journal of Field Archaeology 19(4): 443-470. (in English) (“Cultures néolithiques à 'Ain Ghazal, Jordanie”) doi:10.2307/530427

Schmidt, K. 1994, The Nevali Çori Industry. Status of Research. In: Neolithic Chipped Stone Industries of the Fertile Crescent (Gebel, H.G.K., \& Kozłowski, S.K., Eds.), SENEPSE Vol. 1, Ex Oriente, Berlin: p. 239-251. (in English) (“L’industrie de Nevali Çori. État de la recherche")

Schmidt, K. 2001, Göbekli Tepe, Southeastern Turkey. A Preliminary Report on the 19951999 Excavations. Paléorient, 26(1): 45-54. (in English) (“Göbekli Tepe, Turquie du sud-est. Report préliminaire sur les fouilles de 1995-1999”)

doi:10.3406/paleo.2000.4697

Stordeur, D., \& Abbès F. 2002, Du PPNA au PPNB: mise en lumière d'une phase de transition à Jerf el Ahmar (Syrie), Bulletin de la Société Préhistorique Française, 99(3): 563-595. (in French) ("From PPNA to PPNB: highlighting a transition phase in Jerf el Ahmar”) doi:10.3406/bspf.2002.12712

Stordeur, D., Helmer, D., Jamous, B., Khawam, R., Molist, M., \& Willcox, G. 2010, Le PPNB de Syrie du Sud à travers les découvertes récentes à Tell Aswad. In: La Syrie du Sud du Néolithique à l'Antiquité Tardive. Hauran V, Volume I (Maqdissi, M., Braemer, F., \& Dentzer, J.M., Eds.), Bibliotèque Archéologique et Historique Vol. 191, Institut Français du Proche-Orient, Beirut: p. 41-67. (in French) ("The PPNB of southern Syria through recent discoveries at Tell Aswad")

Tsuneki, A., Arimura, M., Maeda, O., Tanno, K., \& Anezaki, T. 2006, The Early PPNB in the North Levant: A New Perspective from Tell Ain el-Kerkh, Northwest Syria. Paléorient, 32(1): 47-71. (in English) ("Le PPNB ancien dans le nord du Levant : une nouvelle perspective de Tell Ain el-Kerkh, nord-ouest de la Syrie ”) doi:10.3406/paleo.2006.5170

Valla, F., \& Plisson, H. 2005, L'abandon du microlithisme au Levant, fait technique et fait de culture. Journal of the Israel Prehistoric Society, 35: 309-336. (in French) (The abandonment of microlithism, technical fact and cultural fact") URL: http://www.jstor.org/stable/23383567

Wilke, P.J., \& Quintero. L.A. 1994, Naviform Core-and-Blade Technology: Assemblage Character as Determined by Replicative Experiments. In: Neolithic Chipped Stone Industries of the Fertile Crescent (Gebel, H.G.K., \& Kozłowski, S.K., Eds.), SENEPSE Vol. 1, Ex Oriente, Berlin: p. 33-60. (in English) (“Technologie naviforme: caractérisation de l'assemblage a partir de leur réplication expérimentale”) 


\title{
Bidirectional blade technology in the Near East during the Pre-Pottery Neolithic B
}

\author{
Ferran Borrell \\ Consejo Superior de Investigaciones Científicas (CSIC-IMF). Egipcíaques 15, 08001, Barcelona, Spain. \\ Email: silmarils1000@hotmail.com
}

\begin{abstract}
:
Bidirectional blade technology (BBT) has traditionally been considered the most common formal component of Pre-Pottery Neolithic B (PPNB) material culture in the Levant during the $c a .9^{\text {th }}-8^{\text {th }}$ millennia cal. BCE. This paper provides an assessment of the current state of the art of the origins of BBT and its rapid diffusion throughout the Levant during the PPNB. Secondly, it summarizes the different regional and temporal variants (knapping schemes) of the bidirectional blade technologies identified in the region, based both on earlier studies which basically depended on core typology, and on more recent technological approaches oriented to deeply understanding the complete chaîne opératoire of this method of blade knapping. Finally, this comprehensive is also aimed at, hopefully, mitigating potential terminological and conceptual confusion caused by the abundance of studies, from different schools and approaches.

The updated and chronologically refined picture of the origins, diffusion and regional variants of bidirectional technology during the PPNB constitutes a major tool for understanding a wide range of aspects of the first farming communities in the Levant (e.g., social complexity, craft specialization and productivity, inter-site and intra-site social interactions, knowledge transfer, exchange networks, etc.) as well as the Neolithization process of the western wing of the Fertile Crescent itself.

Results confirm that BBT originated in the middle Euphrates valley ca. 8900-8800 cal. BCE. Its appearance constituted a marked change in local lithic traditions towards standardized production of large blades with very specific features (straight, naturally pointed and generally robust) which were transformed into projectiles and, in less numbers, sickle blades, knives and other tools. The marked increase in the size of the projectiles related to bidirectional technology constituted, when compared to Epipalaeolithic and PPNA assemblages of the region (dominated by geometrics and small projectiles such as Khiam points), a remarkable shift in projectile technology. Determining what led those Neolithic communities to produce significant quantities of large and standardized projectiles remains, however, little understood.

The wide distribution in varied socioeconomic contexts (mostly farmers and herders but also hunters and foragers) using different raw materials (obsidian and a wide range of local and non-local flints) and the growing regional and spatial variability observed throughout the Levant suggests that BBT did not require the a priori existence of an economic infrastructure that supported craft specialization. Therefore, it is crucial to rethink whether bidirectional technology could be considered, and under what circumstances, a specialized production.
\end{abstract}


The debate of how bidirectional blade technology diffused is far from being closed and only chronologically refined and multidisciplinary regional approaches will provide further solid evidence to enable us to understand the complex processes (whether if it was cultural or demographic) that remain key to defining the Neolithic developments of the western wing of the Fertile Crescent. From the point of view of the lithics, the successful and rapid expansion through different ecosystems and climate regimes together with the rapid adoption by communities based on different modes of subsistence suggest that the diffusion of BBT was part of a major cultural diffusion rather than simply the successful expansion of a method of knapping that offered certain advantages over previous lithic traditions.

Finally, bidirectional blade technology constituted the most characteristic formal material culture during the PPNB, indicating a strong cultural 'connection' between the northern and southern Levant. After several centuries of predominance and remarkable homogeneity, lithic industries in the Levant changed and bidirectional blade technology declined. Its abandonment started in different circumstances and at different times throughout the region (ca. 7200 cal. BCE in the northern Levant and ca. 6500/6400 cal. BCE in the southern Levant). Such chronological décalage in the abandonment of the most common element of PPNB material culture reveals diverging Neolithic cultural developments from around 72006900 cal. BCE.

Keywords: Near East; Pre-Pottery Neolithic B; lithics; naviform; bidirectional blade technology 